TRANSACTIONS OF THE

AMERICAN MATHEMATICAL SOCIETY

Volume 352, Number 12, Pages 5525-5561

S 0002-9947(00)02666-0

Article electronically published on August 8, 2000

\title{
RIBBON TILE INVARIANTS
}

\author{
IGOR PAK
}

\begin{abstract}
Let $\mathbf{T}$ be a finite set of tiles, and $\mathcal{B}$ a set of regions $\Gamma$ tileable by $\mathbf{T}$. We introduce a tile counting group $\mathbb{G}(\mathbf{T}, \mathcal{B})$ as a group of all linear relations for the number of times each tile $\tau \in \mathbf{T}$ can occur in a tiling of a region $\Gamma \in \mathcal{B}$. We compute the tile counting group for a large set of ribbon tiles, also known as rim hooks, in a context of representation theory of the symmetric group.

The tile counting group is presented by its set of generators, which consists of certain new tile invariants. In a special case these invariants generalize the Conway-Lagarias invariant for tromino tilings and a height invariant which is related to computation of characters of the symmetric group.

The heart of the proof is the known bijection between rim hook tableaux and certain standard skew Young tableaux. We also discuss signed tilings by the ribbon tiles and apply our results to the tileability problem.
\end{abstract}

\section{TRIVIA}

Suppose we are given a set of the tiles on a plane. We are allowed to use translations of the tiles to arrange them in a geometric shape (each tile may occur several times). This arrangement is called tiling of that shape. One can ask whether a given region can be tiled by a given set of tiles, and if it can, how many different tilings there are.

For example, with a set of tiles shown in Figure 0.1 one can make four different tilings of the 4-by-6 rectangle. Two of them are shown in Figure 0.2. Now one can try to find a criterion for when you can tile a rectangle. Observe that each of these tiles alone can tile the whole plane.

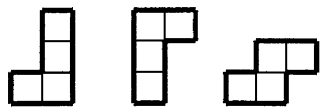

Figure 0.1
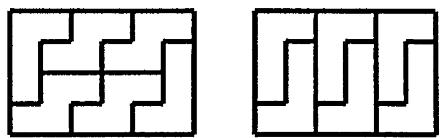

FiguRE 0.2 .

Our personal favorite example is given by the set of tiles shown in Figure 0.3. One can show that there exists only one tiling of the fourth quadrant (see Fig. 0.4). The proof is left to the reader.

It turns out that there are certain nice sets of tiles for which it is not clear whether a given region can be tiled. Here is an example. Consider the 8 tiles shown in Figure 0.5. One can show that the 25-by-25 square cannot be tiled by these tiles.

Received by the editors December 12, 1997.

2000 Mathematics Subject Classification. Primary 05E10, 52C20; Secondary 05B45, 20 C30.

Key words and phrases. Polyomino tilings, tile invariants, Conway group, rim (ribbon) hooks, Young diagrams, Young tableaux, rim hook bijection, symmetric group. 


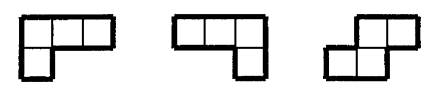

FIGURE 0.3 .

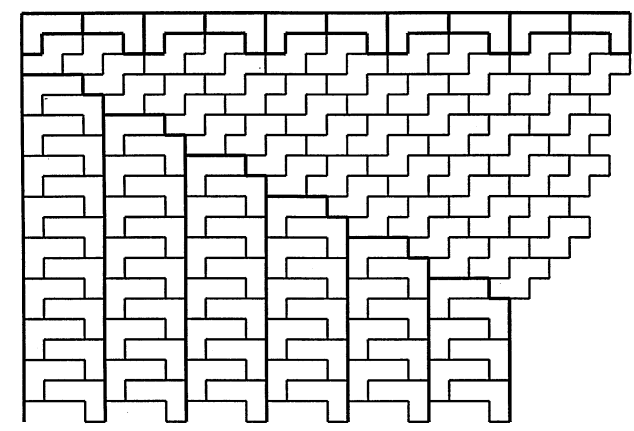

FIGURE 0.4 .

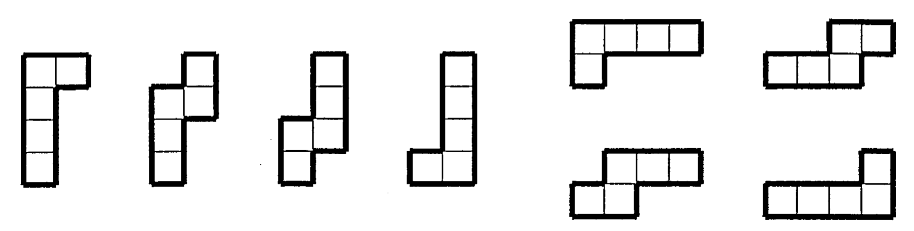

FiguRE 0.5 .

Of course, this could be proved by an exhaustive search. In general, the following result holds.

Theorem 0.1. If an $a-b y-b$ rectangle can be tiled by the tiles shown in Figure 0.5, then $10 \mid a \cdot b$.

Another example. Consider 6 tiles shown in Figure 0.6. We again have

Theorem 0.2. If an $a-b y-b$ rectangle can be tiled by the tiles shown in Figure 0.6, then $10 \mid a \cdot b$.
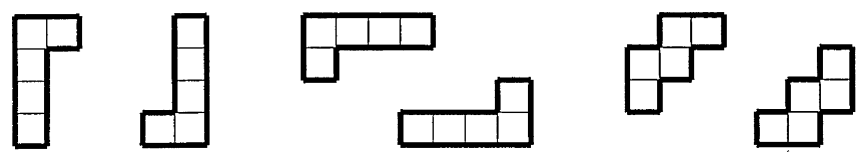

FiguRE 0.6.

Of course, an area argument shows that $5 \mid a \cdot b$.

Consider now a different region. Let $\Delta_{N}$ be a triangular shape as in Figure 0.7. One can check that $\Delta_{24}$ can be tiled by the tiles shown in Figure 0.6 while $\Delta_{25}$ cannot. Generally, 


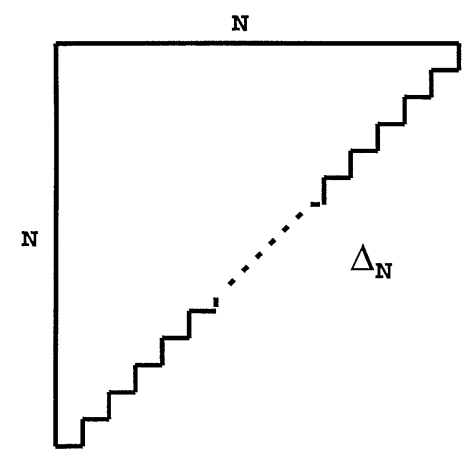

Figure 0.7 .

Theorem 0.3. If $\Delta_{N}$ can be tiled by the tiles shown in Figure 0.6, then $N \equiv$ $0,4,15,19(\bmod 20)$.

It turns out that all three theorems can be proved by use of the same kind of argument. Heuristically, the reason for untileability arises from the following question, completely different in nature:

- Given a set of tiles and a tileable region, are there any linear relations for the number of times each tile occurs in a tiling?

The rest of the paper explains the relevance of this question. All three theorems are proved in section 7 .

\section{INTRODUCTION}

Let $\mathbb{Z}^{2}$ be a square lattice, and $\mathcal{R}$ the set of all compact simply connected regions in $\mathbb{Z}^{2}$. We think of these regions as disjoint unions of $1 \times 1$ squares. Sometimes they are called polyominoes. Fix a finite set of tiles $\mathbf{T}=\left\{\tau_{1}, \ldots, \tau_{N}\right\}, \tau_{i} \in \mathcal{R}$, $i=1, \ldots, N$. Let tiles be invariant under translations. We say that a region $\Gamma \in \mathcal{R}$ is tileable by $\mathbf{T}$ if it can be presented as a disjoint union of the regions

$$
\Gamma=\coprod_{1 \leq j \leq l} \tau_{j}^{\prime},
$$

where each region $\tau_{j}^{\prime}, 1 \leq j \leq l$ is a translation of some $\tau_{i_{j}}$. Such a disjoint union is called a tiling $s$ of $\Gamma$. Denote $\mathcal{S}=\mathcal{S}(\Gamma, \mathbf{T})$ a set of all tilings of $\Gamma$ by the set of tiles $\mathbf{T}$.

Fix a set of tiles $\mathbf{T}$ and a region $\Gamma \in \mathcal{R}$. There are two basic questions one can ask:

- Is $\Gamma$ tileable by $\mathbf{T}$ ?

- If $\Gamma$ is tileable by $\mathbf{T}$, what do the tilings look like?

The first question is very classical and well understood (see e.g. G]). It is usually not hard to find a tiling if $\Gamma$ is tileable by $\mathbf{T}$, while proving the opposite can be extremely difficult. Except for ad hoc examples, there are basically two techniques for proving that a region cannot be tiled: coloring arguments and Conway group analysis (see $[\mathrm{CL},[\mathbf{T}]$ ). Note also that the case when $\mathbf{T}$ contains a 1-by-1 square is trivial: every region is tileable.

While the first questions admits only a "yes" or "no" answer, the second question could be posed in many ways, each of them giving us some information about the 


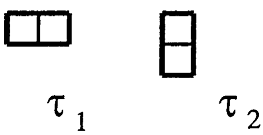

FiguRE 1.1.

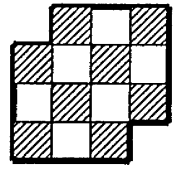

FiguRe 1.2 .

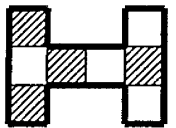

FIGURE 1.3.

structure of the tiling set $\mathcal{S}(\Gamma, \mathbf{T})$. One can find the following two questions in the literature (see e.g. [G], [CEP]):

- How many tilings of $\Gamma$ are there?

- What do random tilings $s \in \mathcal{S}(\Gamma, \mathbf{T})$ look like?

It turns out that the answers to these questions depend heavily on the geometry of $\Gamma$, and can be very complicated even in very simple cases. In particular, finding the number of tilings $|\mathcal{S}(\Gamma, \mathbf{T})|$ is a more general problem than just finding whether a certain region has a tiling. In some cases this problem is known to be NP-complete, and probably cannot be solved by means other than exhaustive enumeration (see [GJ, p. 257]).

To avoid these difficulties we propose another approach to the problem. We fix only $\mathbf{T}$ and ask about properties of tilings of all regions at once. We would like to ask the following two questions:

- Are there any relations for the number of times each tile occurs in a tiling of a given region?

- Is there a finite set of local replacement rules (we also call them local moves or just moves) such that for every region $\Gamma \in \mathcal{R}$, any tiling of $\Gamma$ can be changed into any other tiling by a sequence of moves?

Before we give formal definitions, let us illustrate what happens in the case of dominoes. Although small, this example will illustrate the variety of approaches as well as the complexity of a problem.

Let $\mathbf{T}_{2}$ be a set of two tiles: horizontal domino $\tau_{1}$ and vertical domino $\tau_{2}$ (see Fig. 1.1). It is easy to come up with a necessary condition for tileability (see e.g. G]). Color the region in a checkerboard fashion. Since each domino must contain one black and one white square, the total number of black squares must be equal to the total number of white squares. For example, the region shown in Figure 1.2 cannot be tiled by dominoes since it has 8 black squares and only 6 white squares. Unfortunately, there exist untileable regions with an equal number of black and white squares (see e.g. Fig. 1.3). This means that we need a stronger condition for tileability.

Now suppose we have a region $\Gamma$ that is known to be tileable. We want to compute the number of tilings $\Gamma$ has. This turns out to be an interesting and nontrivial question. In the case of a 2 -by- $m$ rectangle the number of tilings is a Fibonacci number $F(m)=F(m-1)+F(m-2)$ (see Fig. 1.4). In the case of a 


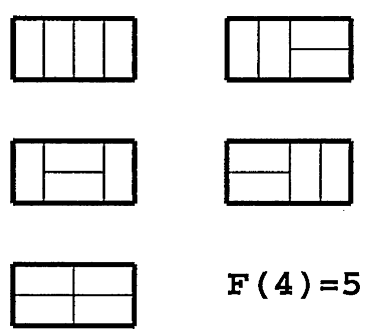

Figure 1.4.

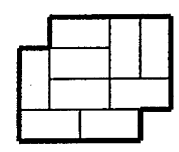

$a_{1}=6$

$a_{2}=3$

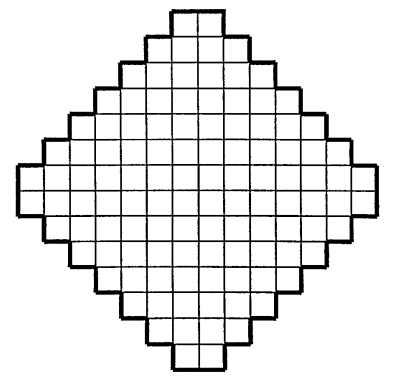

Figure 1.5. Aztec diamond $A_{7}$
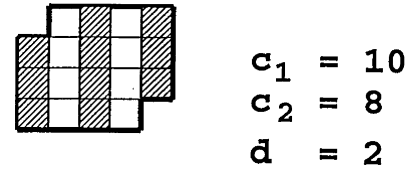

FiguRE 1.7.

general rectangle the problem was solved by Kastelyn and Temperley \& Fisher (see [Ka], TF]). In the case of an Aztec diamond (see Fig. 1.5) the domino tilings were enumerated by Elkies, Kuperberg, Larsen and Propp (see [EKLP). Both results gave rise to many other questions about domino tiling (see e.g. [CEP]). In this work we do not further consider any numerical results of this type.

Let $\Gamma$ be a region tileable by dominoes $\tau_{1}, \tau_{2}$. Consider $s \in \mathcal{S}\left(\Gamma, \mathbf{T}_{2}\right)$, a domino tiling of $\Gamma$. Suppose $s$ consists of $a_{1}=a_{1}(s)$ copies of the horizontal domino $\tau_{1}$, and of $a_{2}=a_{2}(s)$ copies of the vertical domino $\tau_{2}$. Of course, $2\left(a_{1}+a_{2}\right)$ is equal to the area $|\Gamma|$ of the region (see Fig. 1.6). This gives us the first relation. There is one more relation which is less obvious: $a_{2}=$ Const $(\bmod 2)$. To see this, let us color black every other column of the region $\Gamma$ (see Fig. 1.7). Denote by $c_{1}$, $c_{2}$ the number of black and white regions respectively, and put $d=c_{1}-c_{2}$. Since horizontal dominoes contain exactly one black and one white square, and vertical dominoes contain two squares of the same color, we immediately get $a_{2}=d / 2$ $(\bmod 2)($ see Fig. 1.7).

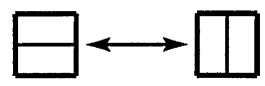

FIGURE 1.8 .

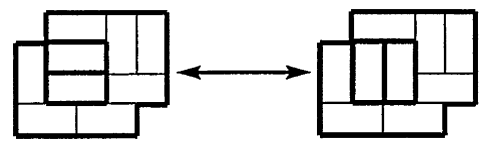

FIGURE 1.9.

Here is another way to look at the set of tilings $\mathcal{S}\left(\Gamma, \mathbf{T}_{2}\right)$. Let us allow the following local replacement rules (or simply moves): take two adjacent horizontal or vertical dominoes and flip them (see Fig 1.8). Of course, this move gives us a new tiling of $\Gamma$ (see Fig 1.9). It is known that by a sequence of such moves we can get from any tiling $s \in \mathcal{S}\left(\Gamma, \mathbf{T}_{2}\right)$ of a simply connected region $\Gamma$ to any other tiling $s^{\prime} \in \mathcal{S}\left(\Gamma, \mathbf{T}_{2}\right)$ (see e.g. [T] $)$. From this we immediately get $a_{1}(s)+a_{2}(s)=$ 
$a_{1}\left(s^{\prime}\right)+a_{2}\left(s^{\prime}\right)$ and $a_{2}(s)=a_{2}\left(s^{\prime}\right)(\bmod 2)$, since these identities are trivial for any single move. This also implies that for a general region there is no other relation for the numbers $a_{1}, a_{2}$ that does not follow from these two. We will use similar logic when proving our main results.

Now we are ready to introduce the tile counting group and tile invariants. Let $\mathbf{T}=\left\{\tau_{1}, \ldots, \tau_{N}\right\}$ be a set of tiles. Denote by $\mathcal{R}_{\mathbf{T}} \subset \mathcal{R}$ set of regions tileable by $\mathbf{T}$. Let $\mathcal{B} \subset \mathcal{R}_{\mathrm{T}}$ be a fixed subset of tileable regions. Consider a tileable region $\Gamma \in \mathcal{B}$. We identify each tiling $s \in \mathcal{S}(\Gamma, \mathbf{T})$ with its multiset of tiles, $s \simeq\left\{\tau_{i_{1}}, \ldots, \tau_{i_{l}}\right\}$. Of course, by doing so we lose some information about the geometric structure of the tilings, since there could be many tilings of $\Gamma$ with the same multiset of tiles (see e.g. Fig. 1.4). As before, by $|\Gamma|$ we denote the area of $\Gamma$.

Let $\mathbb{Z}\langle\mathbf{T}\rangle$ be a group of formal integer linear combinations of $\mathbf{T}$. With each pair of tilings $s_{1}, s_{2} \in \mathcal{S}(T, \Gamma)$ of a region $\Gamma \in \mathcal{B}$ we associate a relation:

$$
\left(\tau_{i_{1}}+\cdots+\tau_{i_{l}}=\tau_{j_{1}}+\cdots+\tau_{j_{r}}\right) .
$$

Let $I$ be the linear span of such relations for all regions $\Gamma \in \mathcal{B}$ and for all pairs of tilings $s_{1}, s_{2} \in \mathcal{S}(T, \Gamma)$. Define the tile counting group to be the quotient group

$$
\mathbb{G}(\mathbf{T} ; \mathcal{B})=\mathbb{Z}\langle\mathbf{T}\rangle / I \text {. }
$$

This will be the main object of our study. Since both groups in the quotient are abelian, one can think of a tile counting group $\mathbb{G}(\mathbf{T} ; \mathcal{B})$ as a subgroup of $\mathbb{Z}\langle\mathbf{T}\rangle$. Thus it is reasonable to describe $\mathbb{G}(\mathbf{T} ; \mathcal{B})$ by its set of independent generators (or the basis) given in $\mathbb{Z}\langle\mathbf{T}\rangle$.

For example, let $\mathbf{T}_{2}$ be a set of dominoes (see Fig. 1.1), and $\mathcal{B}$ a set of simply connected regions. The two tilings in Figure 1.8 correspond to the relation $2 \cdot \tau_{1}=$ $2 \cdot \tau_{2}$. Since every domino tiling of a simply connected region can be obtained from every other domino tiling, $I$ in this case is generated by the above relation. Therefore

$$
\mathbb{G}\left(\mathbf{T}_{2} ; \mathcal{B}\right)=\mathbb{Z}^{2} / I \simeq \mathbb{Z} \times \mathbb{Z}_{2}
$$

The basis can be given as $\tau_{1}+\tau_{2}, \tau_{1}-\tau_{2} \in \mathbb{Z}\left\langle\mathbf{T}_{2}\right\rangle$. Note that the second generator has order 2 as an element in $\mathbb{G}\left(\mathbf{T}_{2} ; \mathcal{B}\right)$, while it has infinite order as an element of $\mathbb{Z}\left\langle\mathbf{T}_{2}\right\rangle$.

Here is another way to describe the tile counting group. Let $G$ be an abelian group, not necessarily finite. A map $f: \mathcal{B} \rightarrow G$ is called a tile invariant (or just an invariant) if for any tileable region $\Gamma \in \mathcal{B}$ and for any tiling $s \in \mathcal{S}(\Gamma, \mathbf{T})$ of it, $s \simeq\left\{\tau_{i_{1}}^{\prime}, \ldots, \tau_{i_{l}}^{\prime}\right\}$, where $\tau_{j}^{\prime}$ is a translation of a tile $\tau_{j}$, we have

$$
f(\Gamma)=f\left(\tau_{i_{1}}\right)+\cdots+f\left(\tau_{i_{l}}\right) .
$$

The problem is to find all the tile invariants for a fixed set of tiles $\mathbf{T}$. Clearly a tile invariant is determined by its values on $\mathbf{T}$, so the problem of finding an invariant is equivalent to finding maps $f: \mathbf{T} \rightarrow G$ which can be extended to the set of all regions $\mathcal{B}$.

Let $\sum_{\tau \in \mathbf{T}} a(\tau) \in \mathbb{Z}\langle\mathbf{T}\rangle$ be an element of a tile counting group $\mathbb{G}=\mathbb{G}(\mathbf{T} ; \mathcal{B})$. Suppose $m$ is its order in $\mathbb{G}$ ( $m$ could be infinity). Then a map $f: \mathbf{T} \rightarrow \mathbb{Z}_{m}$, $m<\infty$, or $f: \mathbf{T} \rightarrow \mathbb{Z}, m=\infty$, defined by $f(\tau)=a(\tau)(\bmod m)$ or $f(\tau)=a(\tau)$, is a tile invariant, where by $\mathbb{Z}_{m}$ we mean the additive group of integers modulo $m$. Conversely, every tile invariant can be lifted to an element of the tile counting group. Thus the problem of computing the tile counting group $\mathbb{G}(\mathbf{T})$ is equivalent to describing all invariants. We say that tile invariants $f_{1}, f_{2}, \ldots$ form an independent 


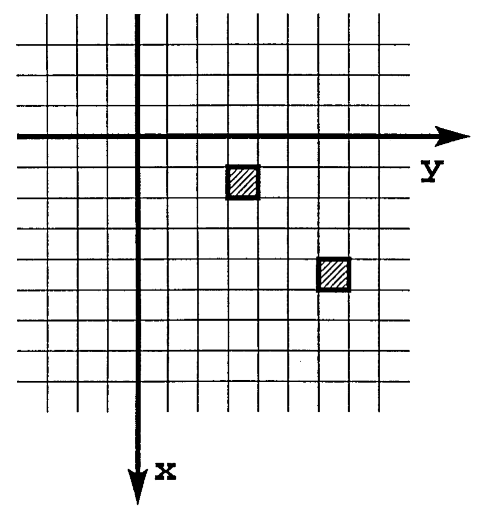

Figure 1.10.

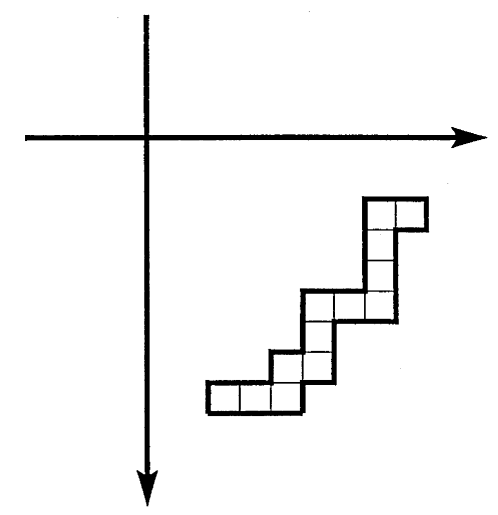

Figure 1.11.

basis of invariants if they correspond to an independent generating set in a tile counting group. An invariant $f: \mathbf{T} \rightarrow G$ is called trivial if $f(\tau)=0$ for all $\tau \in \mathbf{T}$, where $0 \in G$ is the identity element. Otherwise the invariant is called nontrivial. An invariant $f: \mathbf{T} \rightarrow G$ is called primitive if $G \simeq \mathbb{Z}$ or $\mathbb{Z}_{m}$ for some $m$. For the rest of the paper we will be considering only primitive invariants.

Note that when $\mathcal{B}=\mathbf{T}$ every map $f: \mathcal{B} \rightarrow G$ is a tile invariant, i.e $\mathbb{G}(\mathbf{T}, \mathbf{T}) \simeq$ $\mathbb{Z}^{|\mathbf{T}|}$. Generally, the bigger that our set of regions $\mathcal{B}$, the more equations we have on $f$, and the fewer tile invariants we get.

The obvious example of a nontrivial tile invariant is given by the area of tiles:

$$
f_{0}: \mathbf{T} \rightarrow \mathbb{Z}, \quad f_{0}\left(\tau_{i}\right)=\left|\tau_{i}\right|
$$

which can be extended to all tileable regions: $f_{0}(\Gamma)=|\Gamma|$. This implies that the tile counting group has $\mathbb{Z}$ as a subgroup. In the case of domino tiles $\mathbf{T}_{2}$ we also get another invariant (see above):

$$
f_{*}: \mathbf{T}_{2} \rightarrow \mathbb{Z}_{2}, \quad f_{*}\left(\tau_{1}\right)=0, f_{*}\left(\tau_{2}\right)=1 \quad \bmod 2 .
$$

The main result of this paper is a description of a tile counting group for the following set of tiles.

Let the axes on a plane be as shown in Figure 1.10. We say that squares $(i, j)$ and $\left(i^{\prime}, j^{\prime}\right)$ lie on the same diagonal if $i-j=i^{\prime}-j^{\prime}$. For example, the two squares $(2,4)$ and $(5,7)$ lie on the same diagonal (see Fig. 1.10). A ribbon tile is a simply connected region with no two squares lying on the same diagonal. An example of a ribbon tile is shown in Figure 1.11. Denote by $\mathbf{T}_{n}$ the set of all ribbon tiles $\tau$ with $n$ squares: $|\tau|=n$. Obviously, $\mathbf{T}_{2}$ is the set of dominos (see Fig 1.1). The sets $\mathbf{T}_{3}$, $\mathbf{T}_{4}$ and $\mathbf{T}_{5}$ are shown in Figures $1.12-1.14$.

Note that $\left|\mathbf{T}_{n}\right|=2^{n-1}$. Indeed, we can encode each ribbon tile by a sequence $\left(\varepsilon_{1}, \ldots, \varepsilon_{n-1}\right)$ of $n-1$ zeroes and ones as follows. Call the lower left square the starting square. Begin with the starting square and move along the tile. Write $\mathbf{0}$ when going right, and write $\mathbf{1}$ when going up. See Figures $1.12-1.14$ for these coding sequences for all tiles in $\mathbf{T}_{3}, \mathbf{T}_{4}$ and $\mathbf{T}_{5}$. 


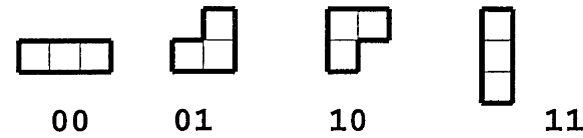

Figure 1.12.

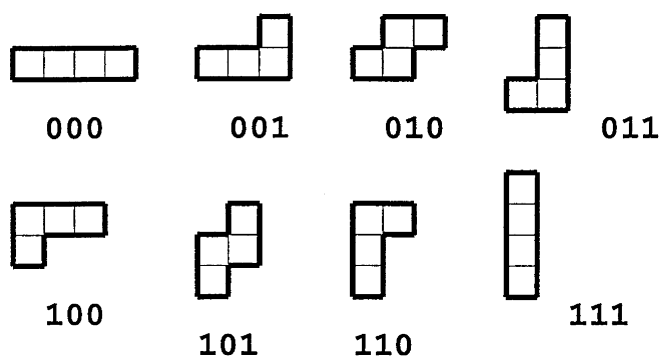

FigURE 1.13.
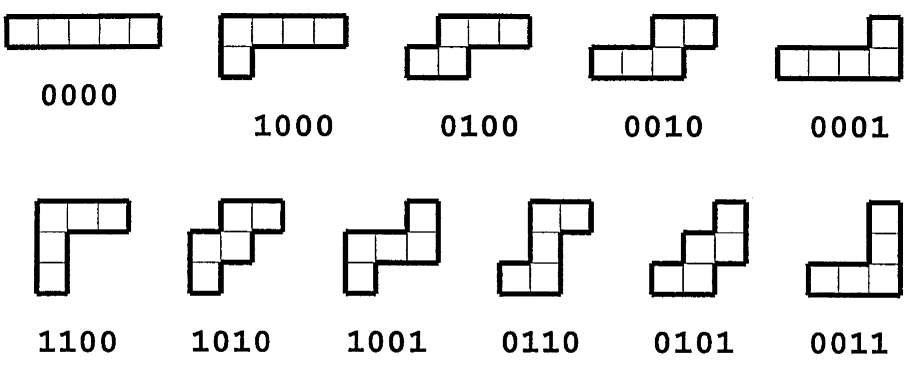

1010

1001

0110

0101

0011
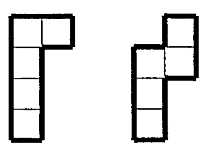

1110

1101
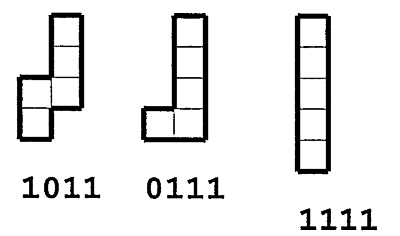

Figure 1.14.

Definition 1.1. Consider the sequence of maps $f_{1}, \ldots, f_{m}: \mathbf{T}_{n} \rightarrow \mathbb{Z}, m=\left\lfloor\frac{n-1}{2}\right\rfloor$, defined as follows:

$$
f_{i}\left(\varepsilon_{1}, \ldots, \varepsilon_{n-1}\right)=\varepsilon_{i}-\varepsilon_{n-i} .
$$

We call the map $f_{i}$ the $i$-convexity invariant.

Definition 1.2. The constant map $f_{0}: \mathbf{T}_{n} \rightarrow \mathbb{Z}$ defined as

$$
f_{0}\left(\varepsilon_{1}, \ldots, \varepsilon_{n-1}\right)=1
$$

is called the area invariant.

Note that the area invariant is designed to be 1 on a tile $\tau \in T_{n}$ rather than $n$. This is designed to simplify the statement of the main result (see below.) 


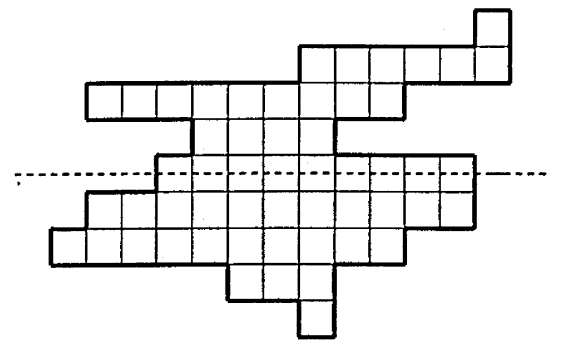

FIGURE 1.15.

Definition 1.3. If $n$ is even, the map $f_{*}: \mathbf{T}_{n} \rightarrow \mathbb{Z}_{2}$ defined as

$$
f_{*}\left(\varepsilon_{1}, \ldots, \varepsilon_{n-1}\right)=\varepsilon_{n / 2} \quad(\bmod 2)
$$

is called the parity invariant.

Before we state our main results, we need to specify the set of regions $\mathcal{B} \in \mathcal{R}_{\mathbf{T}_{n}}$. A region $\Gamma \in \mathcal{R}$ is called row-convex (column-convex) if every horizontal (vertical) line either intersects $\Gamma$ in an interval or does not intersect it at all (see Fig. 1.15). Let $\mathcal{B}_{r c}$ be a set of tileable row-convex simply connected regions. The main result of this paper is the following theorem.

Theorem 1.4. Let $\mathcal{B}=\mathcal{B}_{r c}$ be as above. Then:

1) When $n=2 m+1, \mathbb{G}\left(\mathbf{T}_{n}, \mathcal{B}\right) \simeq \mathbb{Z}^{m+1}$ and the maps $f_{0}, f_{1}, \ldots, f_{m}$ form an independent basis of invariants.

2) When $n=2 m, \mathbb{G}\left(\mathbf{T}_{n}, \mathcal{B}\right) \simeq \mathbb{Z}^{m} \times \mathbb{Z}_{2}$ and the maps $f_{0}, f_{1}, \ldots, f_{m-1}, f_{*}$ form an independent basis of invariants.

When $n=2$ Theorem 1.4 says that the area and parity invariants form an independent basis. Analogously, when $n=3$ Theorem 1.4 says that, aside from the area invariant $f_{0}$, there exists one other nontrivial tile invariant $f_{1}: \mathbf{T} \rightarrow \mathbb{Z}$, where

$$
f_{1}(\mathbf{1 0})=1, \quad f_{1}(\mathbf{0 1})=-1, \quad f_{1}(\mathbf{0 0})=f_{1}(\mathbf{1 1})=0
$$

(see Fig. 1.12). In a different form this invariant was discovered by Conway and Lagarias in [CL] (see also [T]). To say that $f_{1}$ is an invariant is equivalent to saying that:

$$
\# \mathbf{1 0}-\# \mathbf{0 1}=\text { Const. }
$$

This means that the number of times the $\mathbf{1 0}$ tromino occurs in a tiling minus the number of times the $\mathbf{0 1}$ tromino occurs in the same tiling of a region $\Gamma$ depends only on the region $\Gamma$, and not on the tiling.

Here is another nontrivial invariant that exists for all $n>1$.

Definition 1.5. Consider the map $f_{\bullet}: \mathbf{T}_{n} \rightarrow \mathbb{Z}_{2}$ defined as follows:

$$
f_{\bullet}\left(\varepsilon_{1}, \ldots, \varepsilon_{n-1}\right)=\varepsilon_{1}+\varepsilon_{2}+\cdots+\varepsilon_{n-1} \quad(\bmod 2) .
$$

\section{We call $f_{\bullet}$ the height invariant.}

The reason why $f_{\bullet}$ is called the height invariant can easily be seen from the picture. Consider the smallest rectangular box the ribbon tile $\tau$ can fit in (see Fig 1.16). Then $f_{\bullet}(\tau)=a-1(\bmod 2)$, where $a$ is the height of the rectangle. This invariant was considered earlier in connection with certain characters of the 


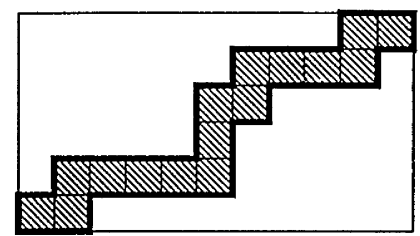

Figure 1.16.

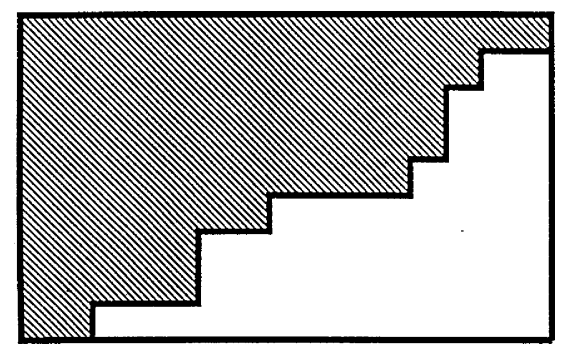

Figure 1.17.

symmetric group $S_{N}$ (see $[\mathbb{R},[\mathrm{JK}],[\underline{\mathrm{S}})$. Namely, it corresponds to signs in the Murnaghan-Nakayama summation formula for computing the character values on the conjugacy classes $\left(n^{a}\right)$, where $N=a \cdot n$ (see [JK], [M] for details). Observe that

$$
f_{\bullet}=\left\{\begin{array}{l}
f_{1}+\cdots+f_{m-1}+f_{m} \quad \bmod 2, n=2 m+1, \\
f_{1}+\cdots+f_{m-1}+f_{*} \quad \bmod 2, n=2 m
\end{array}\right.
$$

This proves that $f_{\bullet}$ is indeed an invariant provided Theorem 1.4 holds.

Now let us say a few words about how Theorem 1.4 is proved. We shall present a finite set of moves which preserve the invariants but enable us to get from any tiling to any other. Formally, let $\mathcal{B}_{y}$ be the set of row- and column-convex regions such that when fit into the smallest possible box they contain the upper right, upper left and the lower left corner of the box (see Fig. 1.17).

Theorem 1.6. Let $\mathcal{B}=\mathcal{B}_{y}$ be as above. For every $n>1$ there is a finite set of at most $n 4^{n}$ moves such that any tiling of $\Gamma \in \mathcal{B}$ by $\mathbf{T}_{n}$ can be transformed by a sequence of moves to any other such a tiling ${ }^{*}$

When $n=2$ we need only one move (see Fig 1.8 ). When $n=3$ we already need 6 moves (see Fig 1.18). Together with Theorem 1.6, we prove this in section 3.

To finish the introduction, let us compare the definition of tile invariants with the generalized coloring arguments introduced by Conway and Lagarias (see [CL]).

Let $G$ be an abelian group, not necessarily finite, and $e$ its identity element. A map $f: \mathcal{R} \rightarrow G$ is called a coloring map if for every region $\Gamma \in \mathcal{R}$ we have

$$
f(\Gamma)=f\left(x_{1}\right)+\cdots+f\left(x_{|\Gamma|}\right),
$$

where $x_{1}, \ldots, x_{|\Gamma|}$ are the squares in $\Gamma$. Of course, $f$ is defined by its values on all $1 \times 1$ squares.

* Ron Adin points out that the minimum number of local moves is exactly $\left(\begin{array}{c}\left|\mathbf{T}_{n}\right| \\ 2\end{array}\right)$. The calculation uses our analysis in section 3. This gives $\left(\begin{array}{l}4 \\ 2\end{array}\right)=6$ moves for $n=3$, and $\left(\begin{array}{l}8 \\ 2\end{array}\right)=28$ for $n=4$. 


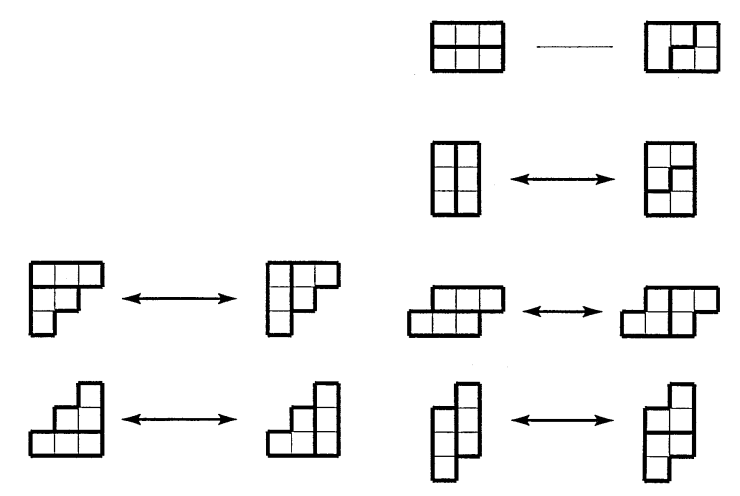

Figure 1.18.

A coloring map $f: \mathcal{R} \rightarrow G$ is called $\mathbf{T}$-coloring if $f(\Gamma)=e$ for all $\Gamma \in \mathcal{R}_{\mathbf{T}}$. In a sense the $\mathbf{T}$-coloring maps are complementary to tile invariants, which are defined only on tileable regions.

It is clear that to show that $f$ is a $\mathbf{T}$-coloring map, all we need is to check that $f\left(\tau^{\prime}\right)=e$ for all translations $\tau^{\prime}$ of a tile $\tau \in \mathbf{T}$. If this is the case, we say that $f$ gives a coloring argument for a set of tiles $\mathbf{T}$. The idea is that we show that now $f(\Gamma)=e$ becomes a necessary condition for $\mathbf{T}$-tileability, which is usually easy to check. Various arguments can be found in $\mathrm{G}$.

To give an example, recall the argument we used to prove untileability by dominoes (see Fig. 1.2). It was, basically, a map $f: \mathcal{R} \rightarrow \mathbb{Z}$, defined by

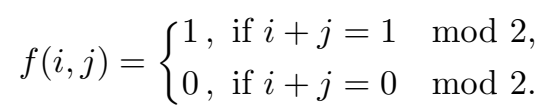

The map $f$ is an example of a $\mathbf{T}$-coloring map. Indeed, observe that, by definition, for both vertical and horizontal dominoes $\tau_{1}, \tau_{2}$ (see Fig. 1.1) we have $f\left(\tau_{1}\right)=$ $f\left(\tau_{2}\right)=0$.

It turns out that coloring arguments cannot be used to prove Theorems $0.1-0.3$. In particular, we have the following result.

Theorem 1.7. Let $\mathbf{T}$ be the set of tiles shown in Figure 0.5. Consider a rectangle $\Gamma=[5 \cdot a \times 5 \cdot b]$, where $a$ and $b$ are odd. Then for any $\mathbf{T}$-coloring map $f: \mathcal{R} \rightarrow G$ we have $f(\Gamma)=e$.

Recall that by Theorem 0.1 the region $\Gamma$ in Theorem 1.7 is not $\mathbf{T}$-tileable. Thus Theorem 0.1 cannot be proved by the use of coloring arguments only. Theorem 1.7 and its analogs for other sets of tiles will be proved in section 8 .

Let us note that coloring maps can be used not only to prove untileability but also to find some tile invariants. Here is how this can be done.

Let $\mathbf{T}$ be a set of tiles, and $G$ an abelian group. Consider a map $g: \mathbf{T} \rightarrow G$. Suppose $f: \mathcal{R} \rightarrow G$ is a coloring map such that $f\left(\tau^{\prime}\right)=g(\tau)$ for all translations $\tau^{\prime}$ of a tile $\tau \in \mathbf{T}$. Then there exists a tile invariant $\widehat{g}: \mathcal{R}_{\mathbf{T}} \rightarrow G$ such that $\widehat{g}(\tau)=g(\tau)$ for all $\tau \in \mathbf{T}$. We call this an extended coloring argument corresponding to the map $f$.

For example, let $\mathbf{T}=\mathbf{T}_{2}$ be the set of dominoes. Consider the coloring map $f: \mathcal{R} \rightarrow \mathbb{Z}_{2}$ defined by $f(i, j)=j \bmod 2$. In a different form the map $f$ was 
considered earlier (see Figure 1.7). We have $g\left(\tau_{1}\right)=1$ and $g\left(\tau_{2}\right)=0$ (see Figure 1.1), which proves that the map $f_{\bullet}$ (see Definition 1.5) is indeed an invariant if $n=2$.

One can try to use the extended coloring argument to get all the tile invariants for a given set of tiles $\mathbf{T}$ and a set of regions $\mathcal{B} \subset \mathcal{R}_{\mathbf{T}}$. It is easy to see that this is impossible if the tile counting group $\mathbb{G}\left(\mathbf{T}_{n}, \mathcal{B}\right) \supsetneq \mathbb{G}\left(\mathbf{T}_{n}, \mathcal{R}_{\mathbf{T}}\right)$, i.e. if there exists a tile invariant for the set of regions $\mathcal{B}$ that is not an invariant for the set of all T-tileable regions $\mathcal{R}_{\mathbf{T}}$. It turns out that in the case of the ribbon tiles $\mathbf{T}_{n}, n>2$, neither convexity nor parity invariants follow from extended coloring arguments. Analogously, for the height invariant we have the following result.

Theorem 1.8. Let $\mathbf{T}_{n}, n>1$, be a set of ribbon tiles. Then the height invariant f. follows from the extended coloring argument if and only if $n=2$.

The proof of Theorem 1.8 is given in section 9. Of course, the "if" part is already proven. Incidentally, proving this theorem was the original goal of this work.

The rest of the paper is constructed as follows. In section 2 we define a rim hook correspondence, which is used in section 3 to prove Theorem 1.6. In section 4 we check that the coloring maps defined in Definitions 1.1-1.3 are invariant under the local moves defined in Theorem 1.5. In section 5 we present a technique for working with tile invariants which enables us to extend the set of regions. In section 6 we show that there are no ribbon tile invariants other than those given in Theorem 1.4. Then we prove the main theorem itself.

The second part of the paper contains several applications of the main result. In section 7 we use ribbon tile invariants to find necessary conditions for tileability. We prove Theorems $0.1-0.3$ and a few other similar results. In section 8 we define and analyze signed tilings and prove Theorem 1.7 along with other related results. This section is motivated by the work [CL] of Conway and Lagarias, although we were able to avoid the use of combinatorial group theory. In section 9 we prove Theorem 1.8 and explore the connection between extended tile arguments and tile invariants. Finally, in section 10 we present several conjectures and open problems.

This work was done while the author was a postdoctoral fellow at MIT. The research was supported by a National Science Foundation Postdoctoral Research Fellowship.

\section{THE RIM HOOK BIJECTION}

Let us recall some standard notation in combinatorics related to the representation theory of the symmetric group (see e.g. [JK], $\mathrm{M}]$ ).

A partition is a nonincreasing integer sequence $\lambda=\left(\lambda_{1}, \lambda_{2}, \ldots, \lambda_{l}\right), \lambda_{1} \geq \lambda_{2} \geq$ $\cdots \geq \lambda_{l}>0$. With each partition $\lambda$ we associate a region $\Gamma_{\lambda}$, called a Young diagram or a Ferrers shape, defined as follows:

$$
\Gamma_{\lambda}=\left\{(i, j) \in \mathbb{Z}^{2} \mid 1 \leq i \leq l, 1 \leq j \leq \lambda_{i}\right\} .
$$

See Figure 2.1 for the Young diagram associated with the partition $(5,5,4,3,1)$. Denote $|\lambda|=\lambda_{1}+\cdots+\lambda_{l}=\left|\Gamma_{\lambda}\right|$.

A skew Young diagram $\Gamma_{\lambda \backslash \mu}$ is the set theoretic difference of the Young diagrams associated with the partitions $\lambda$ and $\mu$ :

$$
\Gamma_{\lambda \backslash \mu}=\Gamma_{\lambda} \backslash \Gamma_{\mu}
$$

For example, the skew Young diagram $\Gamma_{(5,5,4,3,1) \backslash(3,2)}$ is shown in Figure 2.2. 


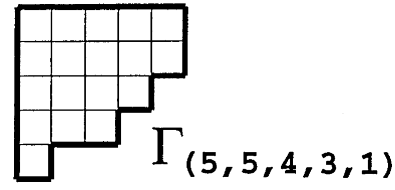

FIGURE 2.1.

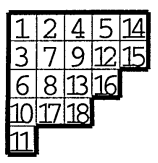

FiguRE 2.3.

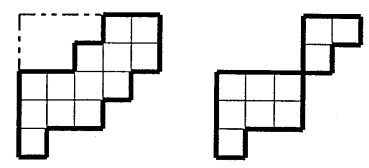

FIGURE 2.2.

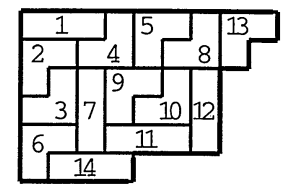

FIGURE 2.4.

To simplify the notation we will use $\lambda$ to denote both the partition and the corresponding region $\Gamma_{\lambda}$, which we also call the Young diagram of shape $\lambda$. By $\mathcal{R}_{y}$ and $\mathcal{R}_{s y}$ we denote the set of all Young diagrams and the set of all skew Young diagrams, respectively.

By $\lambda \circ \mu$ we denote the skew Young diagram obtained as the disjoint union of the Young diagrams $\lambda$ and $\mu$ where $\mu$ is located to the right and above $\lambda$. The example of $(3,3,1) \circ(2,1)=(5,4,3,3,1) \backslash(3,3)$ is shown in Figure 2.2.

A Young tableau is a Young diagram $\lambda$ filled with integer numbers which increase in rows and columns (see Fig. 2.3). A Young tableaux is called standard if these numbers are $1, \ldots,|\lambda|$. We can think of a Young tableau as a flag of Young diagrams $\emptyset=\lambda^{1} \subset \lambda^{2} \subset \ldots \subset \lambda^{n}=\lambda$. A skew Young tableau is defined analogously.

Recall that by $\mathbf{T}_{n}$ we denote a set of ribbon tiles with $n$ squares (see Figs. 1.121.14). A rim hook tableau is a tiling of a Young diagram $\Gamma_{\lambda}$ by ribbon tiles $\tau \in \mathbf{T}_{n}$ filled with numbers $1,2, \ldots,|\lambda| / n$ (squares in the same tile are filled with the same number), and such that squares of tiles with greater numbers are located either to the right or below squares of tiles with smaller numbers (see Fig. 2.4). Again we can think of a rim hook tableau as a flag of Young diagrams.

The rim hook bijection $\varphi$ maps Young diagrams $\lambda,|\lambda|=m \cdot n$, tileable by $\mathbf{T}_{n}$ into $n$-tuples of Young diagrams $\left(\nu^{1}, \ldots, \nu^{n}\right),\left|\nu^{1}\right|+\cdots+\left|\nu^{n}\right|=m$. The bijection $\varphi$ is designed in such a way that whenever we add a ribbon tile to the diagram $\lambda$ on the outside, there exist $i, 1 \leq i \leq n$, such that $\nu^{i}$ gets a square on the outside (see Fig. 2.5). If we think of rim hook tableaux as flag sequences of tileable Young diagrams, the rim hook bijection maps these flag sequences into the $n$-tuples of Young tableaux filled with numbers $1, \ldots, m$ which are increasing in rows and columns in each tableau. It is known that this establishes a bijection between the rim hook tableau of a fixed tileable Young diagram $\lambda$ and the $n$-tuples of Young tableaux with shapes $\varphi(\lambda)=\left(\nu^{1}, \ldots, \nu^{n}\right)$ which are filled with numbers $1, \ldots, m$, where $|\lambda|=m \cdot n$ (see e.g. [JK], [SW]).

The easiest way to understand the rim hook bijection is to look at Figure 2.6. Take a rim hook tableau tiled with 14 tiles $\tau_{i} \in \mathbf{T}_{3}$ and rotate it counterclockwise $135^{\circ}$ degrees. Then project all hooks on the horizontal axis, preserving their labels and relative order. Split the "shadows" into three ( $n$ in the general case) separate sets of "shadows" depending on their horizontal coordinate mod 3. Then simply 


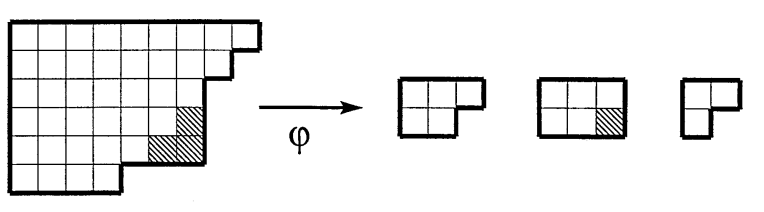

Figure 2.5.

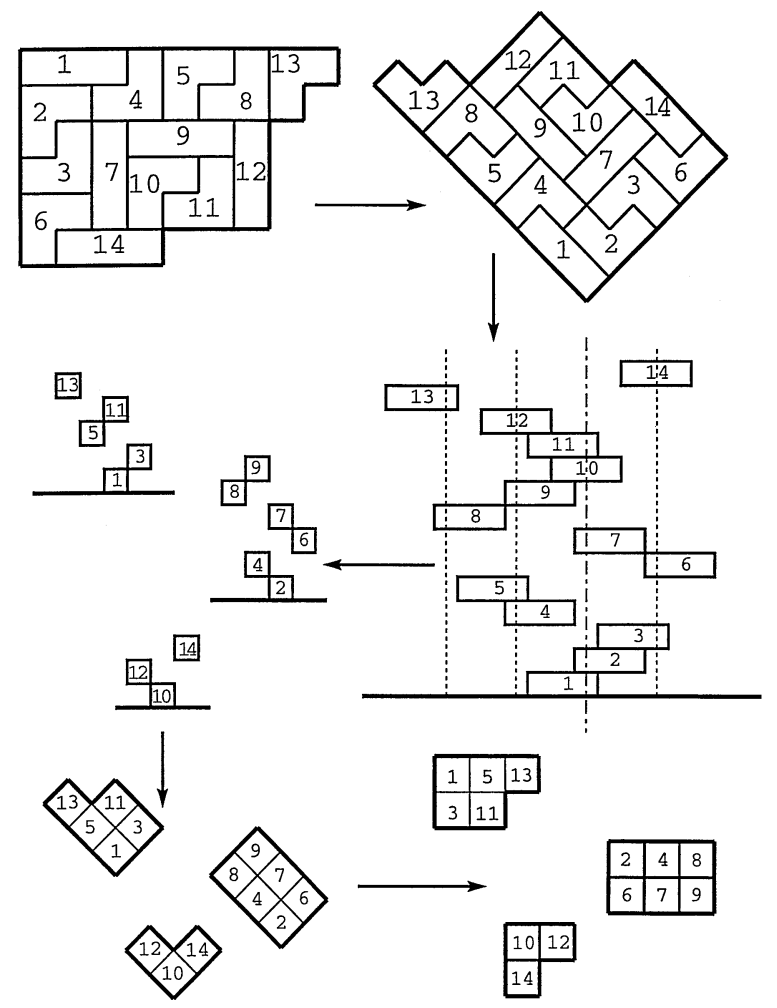

FiguRE 2.6.

shorten the shadows and reverse the procedure. At the end we get three Young tableaux filled with the numbers $1, \ldots, 14$ (see Figure 2.6).

Theorem 2.1. The map $\varphi$ defined above is a one-to-one correspondence.

The theorem goes back to Nakayama and Robinson (see [R], JK]). In modern times it was rediscovered by Stanton and White (see [SW], [FS]) and is sometimes attributed to them.

Another way to think of the rim hook bijection is to say that it establishes a bijection between rim hook tableaux of shape $\lambda$ and standard Young tableaux of the skew shape $\nu^{1} \circ \cdots \circ \nu^{n}$. We shall use this interpretation in the next section. Various proofs and applications of the theorem can be found in [JK], [FS], [ $]$. 


\section{LOCAL MOVES}

Let $\lambda \backslash \mu$ be a skew Young diagram. We define local moves on a set of standard Young tableaux of shape $\lambda \backslash \mu$ as follows. Take a pair of numbers $i$ and $i+1$, $1 \leq i<|\lambda|$, and exchange them if they lie in different rows and columns. We claim that, using these moves, one can start with any standard Young tableau of shape $\lambda \backslash \mu$ and get any other such tableau.

Formally, let $\Omega(\lambda \backslash \mu)$ be a graph with vertices all standard Young tableaux of shape $\lambda \backslash \mu$ and edges obtained by applying local moves. An example with $\lambda=(3,2)$ and $\mu=\emptyset$ is shown in Figure 3.1.

Theorem 3.1. Let $\lambda \backslash \mu$ is a skew Young diagram. Then its graph $\Omega(\lambda \backslash \mu)$ is connected.

This result is known and not hard to prove. Some generalizations and applications can be found in $[\mathrm{BW},[\mathrm{BK}]$.

Sketch of Proof. Introduce an orientation of edges of the graph $\Omega(\lambda \backslash \mu)$ by distinguishing situations when a local move exchanges $i$ and $i+1$ with $i+1$ lying to the right and above $i$ from those where $i+1$ lies to the left and below $i$ (see Figure 3.1 ). Observe that the orientation is acyclic and has exactly one sink. This proves the result.

Consider what happens if we apply the bijection $\varphi$ to the vertices of a graph $\Omega(\lambda \backslash \mu)$ in Theorem 3.1. Fix a skew Young diagram $\nu=\nu^{1} \circ \cdots \circ \nu^{n}$. Define local moves on a set of rim hook tableaux of shape $\lambda=\varphi^{-1}\left(\nu^{1}, \ldots, \nu^{n}\right)$ by the image of the corresponding local moves on a standard skew Young tableaux. Observe that squares in a Young tableau of shape $\nu$ correspond to rim hooks in a rim hook tableau of shape $\lambda$. Thus the corresponding local moves on a set of rim hook tableaux will preserve all the rim hooks except two. Since these rim hooks have consequent labels, together they form a skew shape which has exactly two rim hook tableaux (see Figure 3.2). Note that the two rim hooks may lie far from each other, in which case the local move is just relabeling of their numbers. When $n=1$ this is the only case that occurs.

Now we are ready to state an analog of Theorem 3.1. Denote by $\Omega_{n}(\lambda \backslash \mu)$ a graph with rim hook tableaux as vertices and edges connecting those pairs of tableaux which have the same set of all but two rim hooks.

Theorem 3.2. Let $\lambda$ be a Young diagram tileable by $\mathbf{T}_{n}$. Then its graph $\Omega_{n}(\lambda)$ is connected.

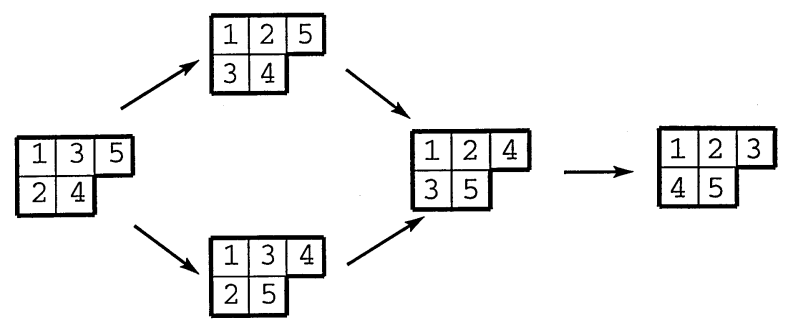

FIGURE 3.1. 


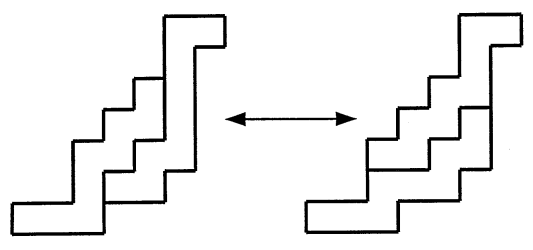

FIGURE 3.2.

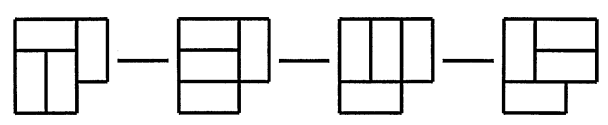

FIGURE 3.3.

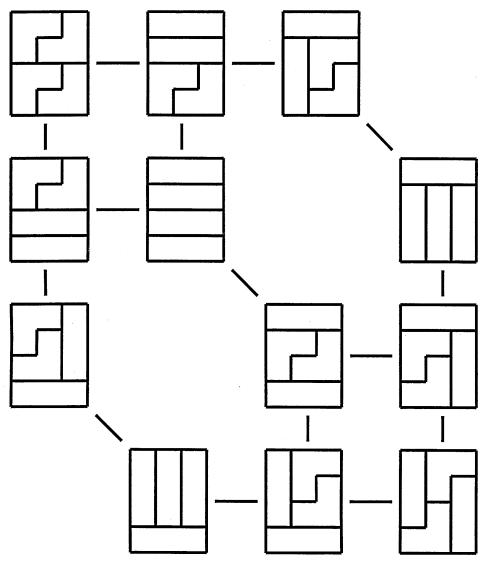

FIGURE 3.4.

Note that here we do not claim that $\Omega_{n}(\lambda \backslash \mu)$ is connected for any tileable skew Young diagram $\lambda \backslash \mu$. It is true and can be proved by the straightforward generalization of the rim hook bijection. We avoid the use of this natural generalization for the purpose of studying ribbon tile invariants.

Proof. Define $\left(\nu^{1}, \ldots, \nu^{n}\right)=\varphi(\lambda)$ to be the image of $\lambda$ under the rim hook correspondence. Consider $G=\Omega\left(\nu^{1} \circ \cdots \circ \nu^{n}\right)$. The correspondence $\varphi^{-1}$ maps vertices of $G$ onto $\Omega_{n}(\lambda)$ and edges onto edges. In other words, $\varphi^{-1}(G)$ is a subgraph of $\Omega_{n}(\lambda)$. By Theorem 3.1 the graph $G$ is connected. Therefore the graph $\Omega_{n}(\lambda)$ is also connected.

Now let us make a graph on the ribbon tilings of $\lambda$. The idea is to erase the labels in the rim hook tableaux and connect those that were connected before.

Formally, consider a graph $\Theta_{n}(\lambda)$ with vertices being all ribbon tilings $s \in$ $\mathcal{S}\left(\Gamma_{\lambda}, \mathbf{T}\right)$ of a fixed shape $\lambda$. Define the edges to be the pairs of tilings that differ by exactly two tiles. Two examples of such graphs $\Theta_{2}(3,3,2)$ and $\Theta_{3}(3,3,3,3)$ are shown in Figure 3.3 and Figure 3.4 respectively.

Theorem 3.3. Let $\lambda$ be a Young diagram tileable by $\mathbf{T}_{n}$. Then the graph $\Theta_{n}(\lambda)$ is connected. 


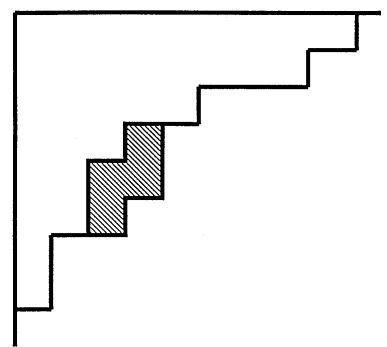

Figure 3.5 .

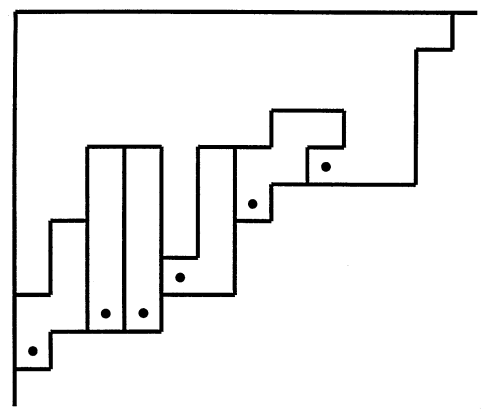

Figure 3.6.

Proof. Consider a map $\iota: \Omega_{n}(\lambda) \rightarrow \Theta_{n}(\lambda)$ which maps rim hook tableaux to ribbon tilings by erasing labels of tiles. By definition $\iota$ maps edges of $\Omega_{n}(\lambda)$ into edges of $\Theta_{n}(\lambda)$. Therefore, in order to prove that $\Theta_{n}(\lambda)$ is connected, all we need to show is that each vertex has a preimage. Indeed, if this is true, in order to find a path between two vertices in $\Theta_{n}(\lambda)$ we simply take their preimages, find a path between them in $\Omega_{n}(\lambda)$, and then map it back to $\Theta_{n}(\lambda)$.

In other words, the theorem in now reduced to the following lemma.

Lemma 3.4. Every ribbon tiling of a Young diagram $\lambda$ admits a labeling which makes it a rim hook tableau of shape $\lambda$.

Proof. We prove the lemma by induction on the number of squares $|\lambda|$. The base case is trivial. Fix a Young diagram $\lambda$. By the border strip of $\lambda$ we mean the set of squares $(i, j) \in \lambda$ such that $(i+1, j+1) \notin \lambda$. A tile $\tau$ is called a border tile if it lies in the border strip (see Figure 3.5). We claim that every ribbon tiling of $\lambda$ must contain at least one border tile. If we find such a tile $\tau$, label it with the largest number. Then there are no tiles that lie to the right of or below $\tau$, and we can proceed by induction with $\lambda \backslash \tau$.

In order to find a border ribbon, start with the lower left corner. It must belong to some tile. This tile has this square as its starting square. If it is not a border tile, find the first border square that is not in that tile. It must belong to some other tile. This tile also has this square as its starting square. Keep on doing so until we find the border tile. It must always exist, since the top right corner must also belong to some tile, and this square cannot be a starting square of any tile unless $n=1$, in which case it is a border tile by definition (see Figure 3.6).

This proves the induction step together with the lemma. The lemma in turn implies Theorem 3.3.

Proof of Theorem 1.6. Observe that the set or regions $\mathcal{B}=\mathcal{B}_{y}$ is exactly the set of all Young diagram shapes. Take the moves to be as described above. The number of different moves is bounded by the the number of pairs of ribbon tiles aligned to each other. The latter number is easily bounded by $n \cdot 4^{n}$, which proves the theorem.

Finally, we would like to note that in the proof of Lemma 3.4 rim hooks need not be of the same length. 


\section{Tile inVARIANTS}

Let $\mathcal{B}=\mathcal{B}_{y}$ be a set of tileable Young diagram shapes. In the previous section we showed that all the tilings of $\lambda \in \mathcal{B}_{y}$ can be obtained from each other by a finite set of moves. Here we show that the maps $f_{i}, 0 \leq i<n / 2$, are constant along these moves. In other words, we shall prove Theorem 1.4 for the set of regions $\mathcal{B}_{y}$.

Let us look at the structure of the moves we introduced in section 3. Consider a large enough example, shown in Figure 3.2. Recall that each ribbon tile is encoded by a sequence $\left(\varepsilon_{1}, \ldots, \varepsilon_{n-1}\right)$ of $n-1$ zeroes and ones. In this notation our pair of tiles is mapped into a similar pair:

\section{$00110101,01011110 \rightarrow 00100101,01010110$.}

Subtracting the sequences as vectors, we get the vectors $(0,0,0,-1,0,0,0,0)$ and $(0,0,0,0,-1,0,0,0)$. In other words, the first tiles in a pair differ at the fourth place, where 1 becomes 0 . Respectively, the second tiles in a pair differ at the fifth place, where 1 again becomes 0 . Note that all tiles contain $n=9$ squares, and $5+4=9$. We claim that this is a general observation.

Lemma 4.1. In every move defined above one tile sequence changes from 1 to 0 (or from 0 to 1 ) at some place $i$ while another has exactly the same change at place $n-i$.

Proof. The proof is done by the following observation. Note that each skew Young diagram shape which corresponds to a move can be broken into three parts by the number of squares in a diagonal parallel to the line $y=-x$ (see Figure 4.1). After the move, the southwest and northeast part remain the same, while the middle part remain divided into two identical small ribbon tiles which get switched now. Therefore, the differences in tile sequences occur only in places where the southwest and northeast parts touch the middle part. If the southwest part was touching the upper of two small ribbon tiles, it now touches the lower one. This means that at place $i$ the number in a sequence changed from 1 to 0 (see Figure 4.1). Respectively, the northeast part was touching the lower of two small ribbon tiles and now is touching the upper one. This means that at place $n-i$ the number in a sequence changed from 1 to 0 .

The second case, when the southwest part was touching the upper of two small ribbon tiles before the move, and the lower tile after the move, is analogous. This proves the lemma.

Now recall the definitions of the convexity invariants:

$$
f_{i}\left(\varepsilon_{1}, \ldots, \varepsilon_{n-1}\right)=\varepsilon_{i}-\varepsilon_{n-i},
$$

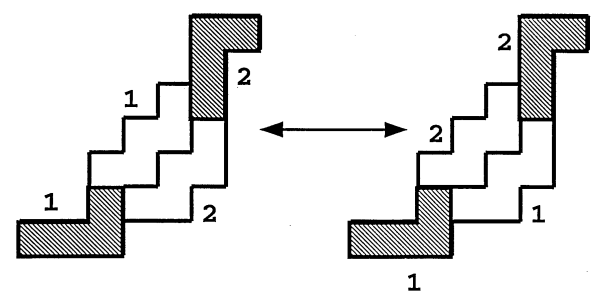

FIGURE 4.1. 
where $1 \leq i \leq\left\lfloor\frac{n-1}{2}\right\rfloor$. Analogously, the parity invariants are defined by

$$
f_{*}\left(\varepsilon_{1}, \ldots, \varepsilon_{n-1}\right)=\varepsilon_{m} \quad \bmod 2,
$$

where $n$ is even and $m=n / 2$.

Lemma 4.2. The maps $f_{i}$ and $f_{*}$ are invariant under the local moves.

Proof. The lemma follows easily from Lemma 4.1. Indeed, as we showed above, if a move changes tiles $\tau_{1}, \tau_{2}$ into tiles $\tau_{1}^{\prime}, \tau_{2}^{\prime}$, then

$$
f_{i}\left(\tau_{1}^{\prime}\right)=f_{i}\left(\tau_{1}\right) \pm 1, \quad f_{i}\left(\tau_{2}^{\prime}\right)=f_{i}\left(\tau_{2}\right) \mp 1,
$$

where the different sign in the second equation comes from the minus sign in $f_{i}=$ $\varepsilon_{i}-\varepsilon_{n-i}$.

Therefore

$$
f_{i}\left(\tau_{1}^{\prime}\right)+f_{i}\left(\tau_{2}^{\prime}\right)=f_{i}\left(\tau_{1}\right)+f_{i}\left(\tau_{2}\right),
$$

which proves that the maps $f_{i}$ are invariant under the local moves.

The case of a parity invariant is slightly different, since here we do not have opposite signs. Instead we have

$$
f_{*}\left(\tau_{1}^{\prime}\right)=f_{*}\left(\tau_{1}\right) \pm 1, \quad f_{*}\left(\tau_{2}^{\prime}\right)=f_{*}\left(\tau_{2}\right) \pm 1 .
$$

Therefore

$$
f_{i}\left(\tau_{1}^{\prime}\right)+f_{i}\left(\tau_{2}^{\prime}\right)=f_{i}\left(\tau_{1}\right)+f_{i}\left(\tau_{2}\right) \pm 2=f_{i}\left(\tau_{1}\right)+f_{i}\left(\tau_{2}\right) \quad(\bmod 2),
$$

which proves that the map $f_{*}$ is invariant under the local moves. This finishes the proof of the lemma.

Recall that by $f_{0}$ we denote the area invariant.

Corollary 4.3. Let $\mathcal{B}=\mathcal{B}_{y}$ be a set of tileable Young diagram shapes. Then, when $n=2 m+1$, the maps $f_{0}, f_{1}, \ldots, f_{m}$ are the ribbon tile invariants. Analogously, when $n=2 m$, the maps $f_{0}, f_{1}, \ldots, f_{m-1}, f_{*}$ are the ribbon tile invariants.

Proof. By Lemma 4.2 the maps $f_{i}$ and $f_{*}$ are invariant under the local moves. By Theorem 3.3 we can get any ribbon tiling of a Young diagram from any other. Therefore these maps are indeed invariants on a set of tileable Young diagram shapes. This proves the corollary.

Note that Corollary 4.3 proves only one part of Theorem 1.4 for the set of regions $\mathcal{B}=\mathcal{B}_{y}$. The second part, which states that these invariants form a basis, will be proven in section 6 .

\section{INCREASING THE SET OF REGIONS}

In this section we will generalize Corollary 4.3 from the set of Young diagram shapes to the set $\mathcal{B}_{r c}$ of all row-convex regions. As an intermediate step we use a set of all skew Young diagram shapes.

Our approach is based on the following general observation.

Lemma 5.1. Let $\mathbf{T}$ be a set of tiles, and let $\mathcal{B}_{1} \subset \mathcal{B}_{2}$ be two sets of $\mathbf{T}$-tileable regions. Suppose for each region $\Gamma_{2} \in \mathcal{B}_{2}$ there is a region $\Gamma_{1} \in \mathcal{B}_{1}$ such that $\Gamma_{1} \supset \Gamma_{2}$ and $\Gamma_{1} \backslash \Gamma_{2}$ is $\mathbf{T}$-tileable. Then, if $f: \mathcal{B}_{2} \rightarrow G$ is an invariant on $\mathcal{B}_{1}$, it is also an invariant on $\mathcal{B}_{2}$. 


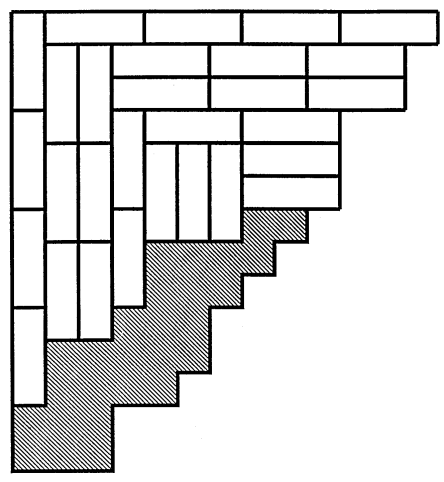

FIGURE 5.1.

Proof. We need to show that for any $\Gamma_{2} \in \mathcal{B}_{2}$ all tilings $s \in \mathcal{S}\left(\Gamma_{2}, \mathbf{T}\right)$ have the same $G$-value of $f$. We know that for any $\Gamma_{2} \in \mathcal{B}_{2}$ there is a region $\Gamma_{1} \in \mathcal{B}_{1}$, $\Gamma_{1} \supset \Gamma_{2}$, such that $\Gamma_{1} \backslash \Gamma_{2}$ is $\mathbf{T}$-tileable. Therefore the set of tilings $\mathcal{S}\left(\Gamma_{2}, \mathbf{T}\right)$ is in a correspondence with a subset $\mathcal{S}^{\prime} \subset \mathcal{S}\left(\Gamma_{1}, \mathbf{T}\right)$ such that all the tilings $s \in \mathcal{S}^{\prime}$ have the same fixed tiling of $\Gamma_{1} \backslash \Gamma_{2}$. By definition the value of $f$ is the same on all tilings of $\mathcal{S}\left(\Gamma_{1}, \mathbf{T}\right)$. Since it is fixed on $\Gamma_{1} \backslash \Gamma_{2}$, it must be the same on $\mathcal{S}\left(\Gamma_{2}, \mathbf{T}\right)$. This finishes the proof.

We call the set $\mathcal{B}_{2}$ of $\mathbf{T}$-tileable regions reducible to $\mathcal{B}_{1}$, if $\mathcal{B}_{1} \subset \mathcal{B}_{2}$ and they satisfy the conditions of Lemma 5.1. Of course, if $\mathcal{B}_{3}$ is reducible to $\mathcal{B}_{2}$ and $\mathcal{B}_{2}$ is reducible to $\mathcal{B}_{1}$, then $\mathcal{B}_{3}$ is reducible to $\mathcal{B}_{1}$.

Lemma 5.2. Let $\mathcal{B}_{\text {sy }}$ be the set of $\mathbf{T}_{n}$-tileable skew Young diagram shapes, and $\mathcal{B}_{y}$ the set of $\mathbf{T}_{n}$-tileable ordinary Young diagram shapes. Then $\mathcal{B}_{\text {sy }}$ is reducible to $\mathcal{B}_{y} \subset \mathcal{B}_{s y}$.

Proof. Indeed, all we need to prove is that every skew Young diagram can be imbedded in an ordinary Young diagram so that their difference is tileable by the ribbon tiles. There is an easy way to do that just by using the horizontal and the vertical tiles.

The idea is shown in Figure 5.1. We start with the rightmost column of a skew Young diagram shape and move to the left. Whenever we move left, add on top a column of vertical tiles until they equal or exceed the column on the right. If they do exceed the column on the right, for each exceeding square add to the right a row of horizontal tiles until they equal or exceed the row below. In example shown in Figure 5.1 we do nothing for the first two columns. For the third column from the right we add one vertical tile and two horizontal. We add just one vertical tile for each of the next two columns. For the sixth column we are forced to add three vertical and two horizontal tiles, etc.

We stop when we are finished with the last column (the ninth in case of Figure 5.1). By construction we always have a Young diagram shape to the right of the building column. Therefore the resulting shape is also a Young diagram shape. This proves the lemma.

Remark 5.3. In $\mathrm{Pa}$ we use this construction to define a generalization of the rim hook bijection for skew shapes. Note also that Lemma 5.2 can be generalized for 


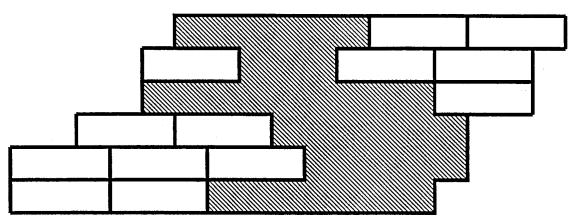

FIGURE 5.2.

any set of tiles $\mathbf{T}$ which contains a horizontal and a vertical tile, not necessarily of the same length.

Lemma 5.4. Let $\mathcal{B}_{\text {sy }}$ be the set of $\mathbf{T}_{n}$-tileable skew Young diagram shapes, and $\mathcal{B}_{r c}$ the set of $\mathbf{T}_{n}$-tileable row-convex regions. Then $\mathcal{B}_{r c}$ is reducible to $\mathcal{B}_{s y} \subset \mathcal{B}_{r c}$.

Proof. Indeed, all we need to prove is that every row-convex region can be imbedded in a skew Young diagram so that their difference is tileable by ribbon tiles. There is an easy way to do that just by using just horizontal tiles.

The idea is shown in Figure 5.2, and is similar to the idea used in Lemma 5.2. We start with the top row of our row-convex region and move to the bottom row. Each time we move down we add a row of horizontal tiles to the left so that they equal or exceed the row above. When get to the bottom we start adding rows of horizontal tiles to the right of the region in such a way that each row equals or exceeds the row below (see Figure 5.2). At the end we get a skew Young diagram shape, which proves the lemma.

Corollary 5.5. The statement of Corollary 4.3 holds for the set $\mathcal{B}_{r c}$ of row-convex $\mathbf{T}_{n}$-tileable regions.

Proof. By Lemmas 5.2 and 5.4, $\mathcal{B}_{r c}$ is reducible to $\mathcal{B}_{y}$. By Lemma 5.1 this implies that every ribbon tile invariant for the set of regions $\mathcal{B}_{y}$ is also an invariant for the set of regions $\mathcal{B}_{r c}$. Together with Corollary 4.3, this proves the result.

\section{The tile Counting Group}

Here we will prove that there are no invariants other than those which follow from convexity, parity and area invariants. Together with Corollary 5.5 this implies the main result of the paper, Theorem 1.4.

The idea is to show that every invariant is completely defined by its values on the horizontal and two-row ribbon tiles.

Denote $\tau_{0}=0000 \ldots 0, \quad \tau_{i}=\mathbf{0} \ldots \mathbf{0 1 0} \ldots \mathbf{0} \quad(\mathbf{1}$ is in the $i$-th place $)$, $1 \leq i \leq n-1$. Let $\mathbf{B}_{n} \subset \mathbf{T}_{n}$ be the set of tiles $\tau_{i}$, where $0 \leq i \leq m=\left\lfloor\frac{n}{2}\right\rfloor$.

Lemma 6.1. Let $f_{1}, f_{2}: \mathcal{B}_{r c} \rightarrow G$ be two ribbon tile invariants. We claim that if for every $\tau \in \mathbf{B}_{n}$ we have $f_{1}(\tau)=f_{2}(\tau)$, then $f_{1} \equiv f_{2}$.

Proof. We need to show that for every ribbon tile $\tau \in T_{n}$ we have $f_{1}(\tau)=f_{2}(\tau)$. This would immediately imply that $f_{1} \equiv f_{2}$. We prove it by induction on the height $h t$ of a ribbon tile (see the Introduction):

$$
h t(\tau)=1+\varepsilon_{1}+\varepsilon_{2}+\cdots+\varepsilon_{n-1} .
$$

First we prove the base of the induction. If $h t(\tau)=1$, then $\tau$ is a horizontal tile $\tau_{0} \in \mathbf{B}_{n}$. If $h t(\tau)=2$, then $\tau \in \mathbf{B}_{n}$ or $\tau=\tau_{i}, m<i \leq n-1$. Observe that $\tau_{i}$ and 


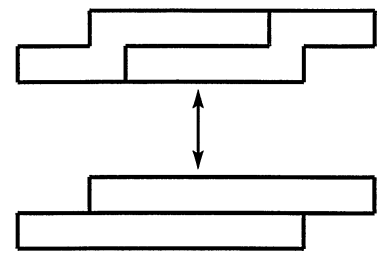

FiguRE 6.1.

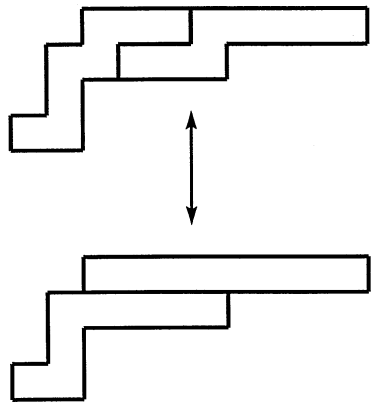

FiguRE 6.2.

$\tau_{n-i}$ form a two-row skew Young diagram shape which can also be divided into two horizontal tiles (see Figure 6.1). Therefore if invariants $f_{1}, f_{2}$ agree on $\mathbf{B}_{n}$ they must also agree on all the two-row ribbon tiles.

Now suppose the claim holds for all tiles $\tau \in \mathbf{T}_{n}$ with $h t(\tau)<k$. Let $\tau \in \mathbf{T}_{n}$ be a ribbon tile and $h t(\tau)=k$. The sequence corresponding to $\tau$ can be presented in the form $\left(\varepsilon_{1}, \ldots, \varepsilon_{i}, 1,0,0, \ldots, 0\right)$. The two-row ribbon tile $\tau_{n-i-1}$ can be aligned with the top two rows of $\tau$ to form a skew Young diagram shape (see Figure 6.2). This region can also be divided into a horizontal tile and a tile $\tau^{\prime}$ with a sequence $\left(\varepsilon_{1}, \ldots, \varepsilon_{i}, 0,0,0, \ldots, 0\right)$ (see Figure 6.2). Note that $h t\left(\tau^{\prime}\right)=h t(\tau)-1=k-1$. Therefore if invariants $f_{1}, f_{2}$ agree on all tiles $\tau \in \mathbf{T}_{n}, h t(\tau)<k$, they must also agree on all tiles $\tau \in \mathbf{T}_{n}, h t(\tau)=k$. This proves the induction step and finishes the proof of the lemma.

Proof of Theorem 1.4. Corollary 5.5 implies that our maps are indeed invariants. All we need to prove now is that they are independent and generate the whole tile counting group.

To show independence, consider values our invariants take on $\mathbf{B}_{n} \subset \mathbf{T}_{n}$. The area invariant $f_{0}$ is a constant on all $\mathbf{B}_{n}$, including $\tau_{0}$. The convexity invariant $f_{i}$ is nonzero only on the tile $\tau_{i}$. Analogously, the parity invariant is nonzero only on the tile $\tau_{m}, n=2 m$. This immediately implies independence.

Now, Lemma 6.1 proves that a tile invariant is completely determined by its values on $\mathbf{B}_{n}$. This implies that when $n=2 m+1$ there can be no invariants that are not generated by $f_{0}, f_{1}, \ldots, f_{m}$. Therefore $\mathbb{G}(\mathbf{T} ; \mathcal{B}) \simeq \mathbb{Z}^{m+1}$.

We still have a little room left when $n=2 m$, since the parity invariant takes values in $\mathbb{Z}_{2}$ rather than in $\mathbb{Z}$. Recall that in the proof of Lemma 6.1 we showed that if $f$ is an invariant, then

$$
f\left(\tau_{i}\right)+f\left(\tau_{n-i}\right)=2 f\left(\tau_{0}\right)
$$

(see Figure 6.1). When $i=m$ this gives $2 \cdot f\left(\tau_{m}\right)=2 \cdot f\left(\tau_{0}\right)$, which implies that there can be no invariants that are not generated by $f_{0}, f_{1}, \ldots, f_{m-1}$ and $f_{*}$. Therefore $\mathbb{G}(\mathbf{T} ; \mathcal{B}) \simeq \mathbb{Z}^{m} \times \mathbb{Z}_{2}$. This finishes the proof of Theorem 1.4

\section{Applications to tileability}

In this section we use ribbon tile invariants to give new tileability criteria for certain sets of tiles. Among the results we prove Theorems $0.1-0.3$. 
We use the following logic. Let $\mathbf{T} \subset \mathbf{T}_{n}$ be a subset of ribbon tiles. Suppose $\Gamma$ is a region which is tileable by $\mathbf{T}_{n}$. Then we can use tile invariants to find diophantine equations for the number of times each tile $\tau \in \mathbf{T}_{n}$ occurs in the tilings. When restricted to a smaller set of tiles $\mathbf{T}$, sometimes these equations do not have an integer solution. This would imply that $\Gamma$ is untileable by $\mathbf{T}$. Generally, having a solution for these equations becomes a tileability test which is easy to use in practice.

Here are a few examples when we apply the above logic successfully.

1. Let $\mathbf{T} \subset \mathbf{T}_{4}$ be the set of four tiles shown in Figure 7.1. We ask for which values $(a, b)$ the rectangle $[a \times b]$ can be tiled by $\mathbf{T}$. Note that since $\mathbf{T}$ is asymmetric, we need to use the following notation: $a$ is a height and $b$ is a width of a rectangle.

Theorem 7.1. Let $\mathbf{T}$ be the set of tiles in Figure 7.1. Then the rectangle $[a \times b]$ can be tiled by $\mathbf{T}$ if and only if $(a, b)$ satisfies one of the following:

1) $4 \mid a$,

2) $8 \mid b, \quad a \geq 3$

3) $2|a, 4| b$.

Proof. The tileability in cases 1) - 3) follows from the existence of tilings of the rectangles $[4 \times 1],[2 \times 4]$ and $[3 \times 8]$ (see Figure 7.2 ).

To prove untileability in all the other cases we need several observations. First, it is obvious that no rectangle $[1 \times b]$ can be tiled.

Suppose now $a$ is odd, $a \geq 3$. Then $4 \mid b$ and the rectangle $[a \times b]$ can be tiled by a horizontal tile $[1 \times 4] \in \mathbf{T}_{4}$. Thus the height invariant (see Definition 1.5) $f_{\bullet}=f_{1}+f_{2}(\bmod 2), f_{\bullet}: \mathcal{B} \rightarrow \mathbb{Z}_{2}$, has the value

$$
f_{\bullet}([a \times b])=0 .
$$

However, $f_{\bullet}(\tau)=1$ for all $\tau \in \mathbf{T}$. Therefore in order to be tileable, the rectangle $[a \times b]$ must contain an even number of tiles, i.e. $8 \mid b$ and we are in case 2).

Now we need to show that no rectangle $[a \times b]$ with $a, b=2 \bmod 4$ can be tiled by $\mathbf{T}$. This can be done by coloring arguments (see the Introduction). We will do it in the next section (see Corollary 8.5).

2. Let $\mathbf{T} \subset \mathbf{T}_{5}$ be the set of eight tiles shown in Figure 0.5. Note that in this case $\mathbf{T}$ is symmetric under the transposition. Theorem 0.1 claims that a rectangle $[a \times b]$ can be tiled by $\mathbf{T}$ only if $10 \mid a \cdot b$. We claim that an even stronger statement is true.

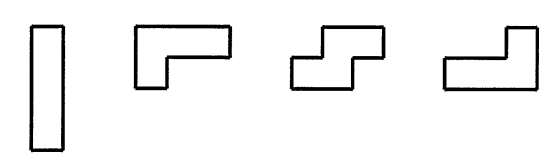

FIGURE 7.1 .

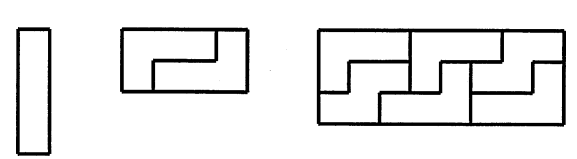

Figure 7.2. 

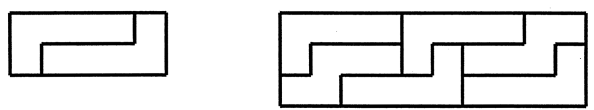

Figure 7.3 .
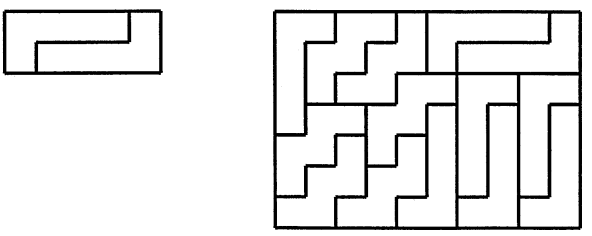

FIGURE 7.4.

Theorem 7.2. Let $\mathbf{T}$ be the set of tiles in Figure 0.5. Then the rectangle $[a \times b]$ can be tiled by $\mathbf{T}$ if and only if $10 \mid a \cdot b$ and $a, b>1$.

Proof. The tileability follows immediately from the existence of tilings of the rectangles $[2 \times 5],[3 \times 10]$ and of the rectangles [5 $\times 2],[10 \times 3]$ transpose to them (see Figure 7.3).

The other direction is similar to the proof of Theorem 7.1. Observe that if a rectangle $[a \times b]$ can be tiled by $\mathbf{T}$, then either $a$ or $b$ can be divided by 5 . But then $[a \times b]$ is tileable by either a horizontal tile $\tau_{0}$ or a vertical tile $\tau_{0}^{\prime}, \tau_{0}, \tau_{0}^{\prime} \in \mathbf{T}_{5}$. Observe that

$$
f_{\bullet}\left(\tau_{0}\right)=f_{\bullet}\left(\tau_{0}^{\prime}\right)=0 \bmod 2,
$$

where $f_{\bullet}: \mathcal{B} \rightarrow \mathbb{Z}_{2}$ is a height invariant. Therefore $f_{\bullet}([a \times b])=0$ for any tileable rectangle $[a \times b]$. On the other hand, for any tile $\tau \in \mathbf{T}$ we have $f_{\bullet}(\tau)=1$. Therefore there must be an even number of tiles in $[a \times b]$, i.e. $10 \mid a \cdot b$. This proves the theorem.

3. Let $\mathbf{T} \subset \mathbf{T}_{5}$ be the set of six tiles shown in Figure 0.6. Note that in this case $\mathbf{T}$ is also symmetric under the transposition.

Theorem 7.3. Let $\mathbf{T}$ be the set of tiles in Figure 0.5. Then the rectangle $[a \times b]$ can be tiled by $\mathbf{T}$ if and only if $10 \mid a \cdot b$ and $a, b \neq 1,3$.

Proof. The tileability follows immediately from the existence of tilings of the rectangles $[2 \times 5],[7 \times 10]$ and of the rectangles $[5 \times 2],[10 \times 7]$ that are transposed to them (see Figure 7.4).

To show the other direction, use the same reasoning as in the proof of Theorem 7.2. If a rectangle $[a \times b]$ can be tiled by $\mathbf{T}$, then its area must be divisible by 5 and therefore it is tileable by either a horizontal or a vertical tile in $\mathbf{T}_{5}$. Thus the 1-convexity invariant $f_{1}$ is 0 on any tileable $[a \times b]$. On the other hand, $f_{1}(\tau)= \pm 1$ for each $\tau \in \mathbf{T}$. Therefore $[a \times b]$ has to be tiled by an even number of tiles, and $10 \mid a \cdot b$. This finishes the proof of the theorem.

Theorem 7.4. The triangular shape $\Delta_{N}$ can be tiled by tiles shown in Figure 0.6 if and only if $N \equiv 0,4,15,19(\bmod 20)$.

Proof. First we prove the tileability part. Observe that $\Delta_{4}$ and $\Delta_{15}$ are both tileable (see Figure 7.5). Now, if we have tilings of the regions $\Delta_{M}, \Delta_{N}$ we can construct a 


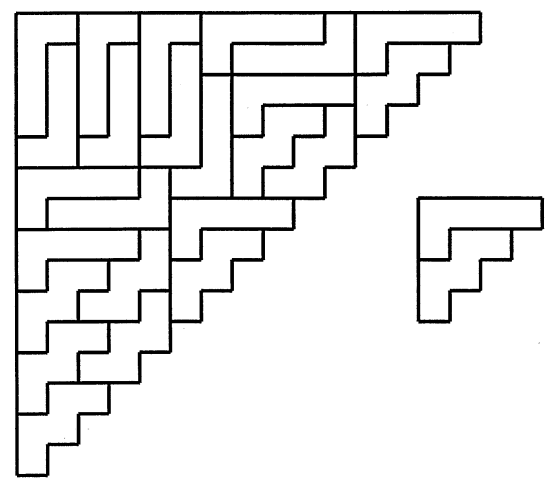

Figure 7.5 .

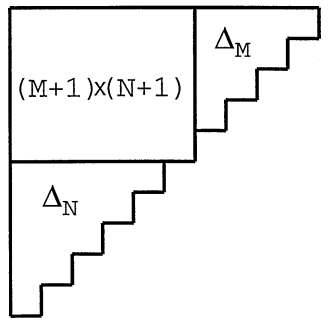

FiguRe 7.6.

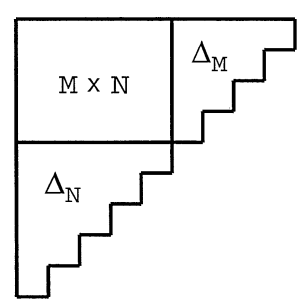

FiguRe 7.7 .
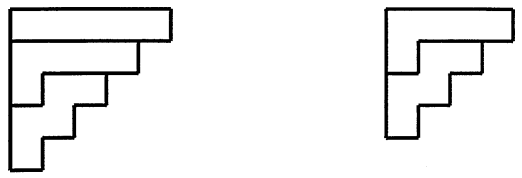

FiguRE 7.8 .

tiling of the region $\Delta_{M+N+1}$ assuming the rectangle $[(M+1) \times(N+1)]$ is tileable. (see Figure 7.6). Analogously, if we have tilings of the regions $\Delta_{M}, \Delta_{N}$ we can construct a tiling of the region $\Delta_{M+N}$ assuming the rectangle $[M \times N]$ is tileable (see Figure 7.7). Since both $[5 \times 16]$ and $[4 \times 15]$ are tileable (see Theorem 7.3), this gives us tilings of $\Delta_{20}=\Delta_{15+5+1}$ and $\Delta_{19}=\Delta_{15+5}$. Going further, if $\Delta_{M}$ is tileable, then $\Delta_{20+M}$ is also tileable. This covers all the values $N \equiv 0,4,15,19$ $(\bmod 20)$.

To prove the "only if" part, start by computing the area. We have

$$
f_{0}\left(\Delta_{N}\right)=\frac{N(N+1)}{2} .
$$

Since the area must be divisible by 5 , this gives us $5 \mid N(N+1)$ and $N \equiv 0,4$ $(\bmod 5)$. Now, in each of these cases it is easy to see that $\Delta_{N}$ can be tiled by $\mathbf{T}_{5}$. Indeed, both $\Delta_{4}$ and $\Delta_{5}$ can be tiled by $\mathbf{T}_{5}$ (see Figure 7.8 ). Since any rectangle $[a \times b]$ is tileable by either a horizontal or a vertical tile in $\mathbf{T}_{5}$, we can use the construction in Figure 7.7 repeatedly and tile $\Delta_{N}$ for all $N \equiv 0,4(\bmod 5)$. 

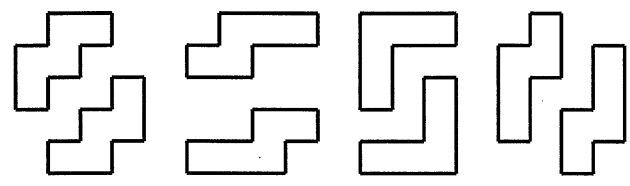

FiguRe 7.9 .
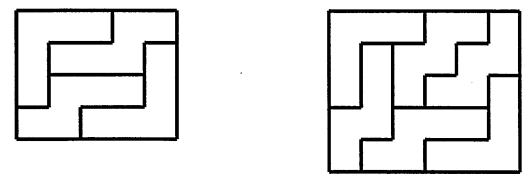

FiguRE 7.10.

Now we can compute the value of the 1-convexity invariant $f_{1}$ on $\Delta_{N}, N \equiv 0,4$ $(\bmod 5)$. We have

$$
f_{1}\left(\Delta_{4}\right)=f_{1}\left(\Delta_{5}\right)=0, f_{1}\left(\Delta_{N+5}\right)=f_{1}\left(\Delta_{N}\right),
$$

which gives us

$$
f_{1}\left(\Delta_{5 m+4}\right)=f_{1}\left(\Delta_{5 m+5}\right)=0 .
$$

Since $f_{1}$ takes only the values \pm 1 on $\mathbf{T}$, this implies that in order to be tileable $\Delta_{N}$ must have an even number of tiles. Thus $2 \mid f_{0}=\frac{N(N+1)}{10}$, and $20 \mid N(N+1)$. This immediately implies $N \equiv 0,4,15,19(\bmod 20)$.

4. Let $\mathbf{T} \subset \mathbf{T}_{5}$ be the set of eight tiles shown in Figure 7.9. As before, we solve the tileability problem for rectangular and triangular shapes.

Theorem 7.5. Let $\mathbf{T}$ be the set of tiles in Figure 7.9. Then a rectangle $[a \times b]$ can be tiled by $\mathbf{T}$ if and only if $(a, b), a \leq b$, satisfies one of the following:

1) $10 \mid a \cdot b, a \geq 8$

2) $a=4,6,5 \mid b$,

3) $a=5,2 \mid b$.

Proof. The tileability follows from the existence of tilings of the rectangles $[4 \times 5]$, $[5 \times 6]$ and of the rectangles $[5 \times 4],[6 \times 5]$ that are transposed to them (see Figure 7.10). Indeed, then we can construct rectangles $[4 \times 5 \mathrm{~m}],[6 \times 5 \mathrm{~m}],[5 \times 2 r], r \geq 3$, which cover cases 2 ) and 3 ). An easy check shows that this also implies tileability in case 1).

Now let us prove the "only if" direction. First, we show that 10 must divide $a b$ in order for $[a \times b]$ to be tileable by $\mathbf{T} \subset \mathbf{T}_{5}$. Indeed, the area $f_{0}=a \cdot b$ must be divisible by 5 , which implies that $[a \times b]$ is tileable by either a horizontal or a vertical tile in $\mathbf{T}_{5}$. Now this implies that the 2 -convexity invariant takes the value zero on a rectangle, while its value is \pm 1 on each of the eight tiles in $\mathbf{T}$. Therefore $[a \times b]$ must contain an even number of tiles in order to be tileable by $\mathbf{T}$, which proves the claim.

Observe that since $10 \mid a \cdot b, a, b>7$, covers by case 1 ) we are left with the cases when $a$ or $b$ is at most 7 . It is easy to see that there are no tileable rectangle with $a=2,3$. An elaborate search of about a dozen beginnings shows that there are no 


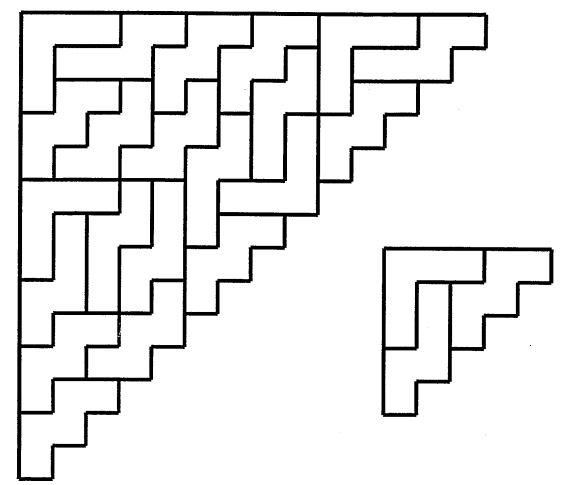

FiguRe 7.11.

tileable rectangle with $a=7$. These cover all the untileable cases, and finishes the proof of the theorem.

Theorem 7.6. The triangular shape $\Delta_{N}$ can be tiled by tiles shown in Figure 7.9 if and only if $N \equiv 0,5,14,19(\bmod 20)$.

Proof. First we prove the tileability part. Observe that $\Delta_{5}$ and $\Delta_{14}$ are both tileable (see Figure 7.11). Recall that by Theorem 7.5 the rectangles $[14 \times 5],[15 \times 6]$ and $[20 \times N], N \geq 5$, are all tileable by $\mathbf{T}$. Using the same construction as in the proof of Theorem 7.4 (see Figures 7.6 and 7.7), we get tilings on $\Delta_{19}, \Delta_{20}$. We also get a tiling of $\Delta_{20+N}$ from a tiling of $\Delta_{N}$. This covers all the values $N \equiv 0,5,14,19$ $(\bmod 20)$ and proves the "if" part.

To prove the "only if" part, first recall that the area is divisible by 5 if and only if $N \equiv 0,4(\bmod 5)$, and in all these cases $\Delta_{N}$ is tileable by $\mathbf{T}_{5}$ (see the proof of Theorem 7.4). Compute the 2-convexity invariant $f_{2}\left(\Delta_{N}\right)$. We have (see Figures 7.7 and 7.8$)$

$$
f_{2}\left(\Delta_{4}\right)=f_{2}\left(\Delta_{5}\right)=-1, \quad f_{2}\left(\Delta_{N+5}\right)=f_{2}\left(\Delta_{N}\right)-1,
$$

which gives us

$$
f_{2}\left(\Delta_{5 m+4}\right)=f_{2}\left(\Delta_{5 m+5}\right)=-m .
$$

Since $f_{2}$ takes only the values \pm 1 on $\mathbf{T}$, this implies that in order to be tileable the number of tiles in $\Delta_{5 m+4,5}$ must have the same parity as $m$. This immediately implies $N \equiv 0,4,15,19(\bmod 20)$, and finishes the proof.

\section{Signed tilings And COLORING ARguments}

Instead of ordinary tilings one can try using signed tilings (see [CL), which are basically placements of tiles on a plane with weights +1 or -1 assigned to each of them. We say that they tile a region if the sum of the weights of the tiles is 1 for every square inside a region and 0 elsewhere.

Even with small sets of tiles it often happens that there are untileable regions which have signed tilings. For example, the region in Figure 1.3 has no ordinary domino tiling but has a signed domino tiling. Indeed, simply tile the rectangle $[3 \times 4]$ by dominoes and add two horizontal dominoes on the top and on the bottom with negative signs. 
It turns out that signed tilings are easier to study because of their connection with coloring arguments. By analogy with tile invariants, a coloring map $f: \mathcal{R} \rightarrow G$ is trivial if $f(\Gamma)=0$, for every $\gamma \in \mathcal{R}$, where 0 is an identity element in an abelian group $G$. Otherwise $f$ is called nontrivial.

Theorem 8.1. A region $\Gamma$ has a signed tiling if and only if there is no abelian group $G$ that has a nontrivial $\mathbf{T}$-coloring map $f: \mathcal{R} \rightarrow G$.

Proof. Indeed, consider a group $\mathfrak{G}$ obtained as a free abelian group generated by elements $x_{i, j}$ that correspond to squares of a square grid. Let $\mathfrak{I} \subset \mathfrak{G}$ be a subgroup generated by sums $x_{i_{1}, j_{1}}+x_{i_{2}, j_{2}}+\ldots$ that correspond to translations of tiles.

Observe that the $\mathbf{T}$-coloring maps correspond to the elements of the quotient group $\mathfrak{G} / \mathfrak{I}$. Therefore for every region $\Gamma$ there exists a $\mathbf{T}$-coloring map $f: \mathcal{R} \rightarrow G$ such that $f(\Gamma) \neq 0$ unless the sum of squares of $\Gamma$ lies in $\mathfrak{I}$. On the other hand, a region $\Gamma$ has a signed tiling if and only if the sum of its squares lies in $\mathfrak{I}$. This proves the result.

By analogy with the tile counting group we define a coloring group $\mathbb{O}(\mathbf{T})=\mathfrak{G} / \mathfrak{I}$. It follows from the proof of the theorem that signed tilings and $\mathbf{T}$-coloring maps are basically dual to each other. However, it is convenient to separate them, since they give a different view of the subject.

It is important to note that whenever we have a $\mathbf{T}$-coloring map which proves that a certain region $\Gamma$ is untileable, this also implies that $\Gamma$ has no signed tiling. Therefore in the event when $\Gamma$ is untileable but has a signed tiling (like the region in Figure 1.3 mentioned above), it also means that this fact cannot be proved by use of $\mathbf{T}$-coloring maps. Traditionally the coloring maps were a major instrument in proving untileability results (see $[\mathrm{G}]$ ), so it is often desirable to check whether they can be used to prove any of our negative results. Below we will show that the results we obtained in the previous section in fact cannot be proved by use of coloring arguments.

Finally, let us point out where the difference between signed and ordinary tilings comes from. Instead of an abelian group $G$ one can take a monoid $M$ (commutative semigroup with an identity element). One can also define a semigroup morphism from all regions to $M$ which is the identity on all tiles. It is not hard to prove a theorem similar to Theorem 8.1 which says that a region $\Gamma$ has a tiling if and only if there exists no morphism which is not the identity on $\Gamma$ (cf. [CL]). We skip the details.

Theorem 8.2. Let $\mathbf{T}_{n}$ be a set of ribbon tiles, and let $\mathbb{O}\left(\mathbf{T}_{n}\right)$ be its coloring group. Then

$$
\mathbb{O}\left(\mathbf{T}_{n}\right) \simeq \mathbb{Z}^{n-1}
$$

Proof. Let $Z$ be an abelian group generated by elements $z_{0}, z_{1}, \ldots, z_{n-1}$. Define a coloring map

$$
\zeta: \mathcal{R} \rightarrow Z /\left(z_{0}+\cdots+z_{n-1}\right),
$$

which acts on generators as follows:

$$
\zeta\left(x_{i, j}\right)=z_{(j-i \bmod n)}
$$

To prove that $\zeta$ is indeed a $\mathbf{T}_{n}$-coloring map, note that by definition ribbon tiles in $\mathbf{T}_{n}$ contain no two squares lying on the same diagonal. Therefore they must 
contain squares lying in $n$ subsequent diagonals: $i, i+1, \ldots, i+n-1$, where $i \in \mathbb{Z}$. Thus for all $\tau \in \mathbf{T}_{n}$

$$
\zeta(\tau)=z_{i}+\cdots+z_{n}+z_{1}+\cdots+z_{i-1}=0
$$

and $\zeta$ is indeed a $\mathbf{T}_{n}$-coloring map.

We can now prove that the coloring group for the set of ribbon tiles $\mathbf{T}_{n}$ is $\mathbb{Z}^{n-1}$. Let us prove that for any region $\Gamma, \zeta(\Gamma)=0$, there exist a signed tiling of $\Gamma$. Then, by Theorem 8.1, this will imply that the coloring group is isomorphic to $Z /\left(z_{0}+\cdots+z_{n-1}\right) \simeq \mathbb{Z}^{n-1}$, which proves the result.

Let $d_{1}$ be the difference of ribbon tiles $00 \ldots 01$ and $00 \ldots 00$ which have the same starting square. Observe that adding $d_{1}$ to a region adds a square $x_{i, j}$ and subtracts a square $x_{i+1, j+1}$ lying on the same diagonal. Let $d_{2}$ be the difference of two horizontal tiles $00 \ldots 0$, one of them having the starting square $x_{i, j}$ and the other in $x_{i, j+1}$. By analogy with $d_{1}, d_{2}$ adds a square $x_{i, j}$ and subtracts a square $x_{i, j+n}$ lying on the same diagonal modulo $n$. Note that all the differences $d_{1}$ and $d_{2}$ are equivalent up to translation.

Now, let $\Gamma$ be a region such that $\zeta(\Gamma)=0$. We show that by adding enough differences $d_{1}$ and $d_{2}$ we can get an empty region. Indeed, by using $d_{2}$ 's we can move all the squares of $\Gamma$ into the first $n$ diagonals. Furthermore, by using $d_{2}$ 's we can move all the squares of $\Gamma$ into the squares $x_{0,0}, x_{0,1}, \ldots, x_{0, n-1}$. Since $\zeta(\Gamma)=0$, this means that we get the same weight $m$ at each of these squares. Now subtract $m$ copies of the horizontal tile. We get an empty region, which is exactly what we needed. This finishes the proof of the theorem.

Note that if we use only $d_{1}$ without $d_{2}$ we get a sequence of numbers $\ldots, m_{-1}$, $m_{0}, m_{1}, m_{2}, \ldots$, which are numbers of squares in diagonals. This sequence gives rise to a coloring argument for the ordinary tilings that is more general than the $\mathbf{T}_{2}$ coloring map $\zeta$. For example, it proves that the region in Figure 1.3 is untileable by dominoes. Indeed, the sequence is $1,1,2,2,2,1,1$. The first two ones imply that there is a domino lying in the first two diagonals. Now, the two in the third place implies that there must be two dominoes that lie in the the third and fourth diagonal. Consequently, there must be two dominoes that lie in the the fifth and sixth diagonal. But this is impossible since the sixth number in the sequence is one. Therefore the region in Figure 1.3 is indeed untileable by dominoes.

When we restrict our set of tiles to a subset, all the coloring maps remain. However, a priori other coloring maps may appear. We show that in the cases considered in the previous section this does not happen.

Theorem 8.3. For the set of tiles shown in Figure 7.1 the coloring group is $\mathbb{Z}^{3}$. For the sets of tiles shown in Figures 0.5, 0.6 and 7.9 the coloring group is $\mathbb{Z}^{4}$.

Proof. All we need to show is that our sets of tiles generate differences $d_{1}$ and $d_{2}$. This would imply that their coloring group is the same as that of the ribbon tiles.

1) For the set of tiles in Figure 7.1, $d_{1}$ comes from subtracting 010 and 001 with the same starting square. The difference $d_{2}$ comes from subtracting $\mathbf{1 0 0}$ and $\mathbf{0 0 1}$ with starting squares $x_{i, j}$ and $x_{i-1, j}$ respectively.

2) For the set of tiles in Figure 0.5, $d_{1}$ comes from subtracting 0010 and 0001 with the same starting square. The difference $d_{2}$ comes from subtracting 0111 and 1110 with starting squares $x_{i, j}$ and $x_{i, j+1}$ respectively.

3) For the set of tiles in Figure 0.6, denote $d_{2}$ the difference between 0111 and 1110 with starting squares $x_{i, j}$ and $x_{i, j+1}$ respectively. It adds $x_{i, j}$ and subtracts 
$x_{i-3, j+2}$. Analogously define a difference $d_{2}^{\prime}$ between 0001 and 1000 with starting squares $x_{i-1, j}$ and $x_{i, j}$ respectively. It subtracts $x_{i, j}$ and adds $x_{i-2, j+3}$. Now the difference $d_{1}$ comes from adding $d_{2}$ and $d_{2}^{\prime}$.

4) For the set of tiles in Figure 7.9, $d_{1}$ comes from subtracting 0010 and $\mathbf{0 0 1 1}$ with the same starting square. The difference $d_{2}$ comes from subtracting 0010 and 0100 with starting squares $x_{i, j}$ and $x_{i, j+1}$ respectively.

Theorem 8.3 basically tells us that $\zeta(\Gamma)=0$ is a criterion for a region $\Gamma$ to have a signed tiling in each of those cases. Before we can conclude, we need the following technical result.

Lemma 8.4. 1) Let $n=5, \Gamma=\Delta_{N}$. Then $\zeta(\Gamma)=e$ if and only if $N \equiv 0,4$ $(\bmod 5)$.

2) Let $n=5, \Gamma=[a \times b]$. Then $\zeta(\Gamma)=e$ if and only if $5 \mid a \cdot b$.

3) Let $n=4, \Gamma=[a \times b]$. Then $\zeta(\Gamma)=e$ if and only if $4 \mid a$ or $4 \mid b$.

Proof. Parts 1) and 2) follow immediately from the area being divisible by 5 and existence of tilings by $T_{5}$ in all these cases (see the proof of Theorem 7.4).

Part 3$)$ is analogous except for the case when $a, b \equiv 2(\bmod 4)$. In this case a simple direct computation of zeta shows that $\zeta \neq e$.

Corollary 8.5. 1) Let $n=5$, and let $\mathbf{T}$ be any of the sets of tiles shown in Figures 0.5, 0.6 and 7.9. Then $\Delta_{N}$ has a signed tiling by $\mathbf{T}$ if and only if $N \equiv 0,4(\bmod 5)$.

2) Let $n=5$, and let $\mathbf{T}$ be any of the sets of tiles shown in Figures 0.5, 0.6 and 7.9. Then $[a \times b]$ has a signed tiling by $\mathbf{T}$ if and only if $5 \mid a \cdot b$.

3) Let $n=4$, and let $\mathbf{T}$ be the set of tiles shown in Figure 7.1. Then $[a \times b]$ has a signed tiling by $\mathbf{T}$ if and only if $4 \mid a$ or $4 \mid b$.

Proof. This follows immediately from Lemma 8.4 and Theorems 8.3 and 8.1.

Corollary 8.6. None of Theorems $0.1-0.3$ can be proved by the coloring arguments.

\section{EXTENDED COLORING ARGUMENTS AND TILE INVARIANTS}

Recall the definition of a coloring group:

$$
\mathbb{O}(\mathbf{T})=\mathfrak{G} / \mathfrak{I}
$$

where $\mathfrak{G}$ is a free abelian group generated by elements $x_{i, j}$ that correspond to squares of a square grid, and let $\mathfrak{I} \subset \mathfrak{G}$ be a subgroup generated by sums $x_{i_{1}, j_{1}}+$ $x_{i_{2}, j_{2}}+\ldots$ that correspond to translations of tiles.

By analogy, define an extended coloring group as follows:

$$
\overline{\mathbb{O}}(\mathbf{T})=\mathfrak{G} / \mathfrak{I}^{\prime}
$$

where $\mathfrak{I}^{\prime} \subset \mathfrak{G}$ is a subgroup generated by relations

$$
x_{i_{1}, j_{1}}+x_{i_{2}, j_{2}}+\cdots=x_{p_{1}, q_{1}}+x_{p_{2}, q_{2}}+\ldots
$$

where the tiles $\tau_{1}=\coprod_{k}\left(i_{k}, j_{k}\right), \tau_{2}=\coprod_{k}\left(p_{k}, q_{k}\right)$ are translations of the same tile $\tau \in \mathbf{T}$. Of course, $\mathbb{O}(\mathbf{T}) \subset \overline{\mathbb{O}}(\mathbf{T})$.

By definition, every extended coloring argument for a set of tiles $\mathbf{T}$ corresponds to an element of the extended coloring group $\overline{\mathbb{O}}(\mathbf{T})$. In other words, there is a map $\nu: \overline{\mathbb{O}}(\mathbf{T}) \rightarrow \mathbb{G}(\mathbf{T})$. Since $\nu$ is homomorphic, the image $\mathbb{E}(\mathbf{T})=\nu(\overline{\mathbb{O}}(\mathbf{T}))$ is a subgroup in the tile counting group $\mathbb{G}(\mathbf{T})$. Call this image $\mathbb{E}(\mathbf{T}) \subset \mathbb{G}(\mathbf{T})$ a torsion group. 


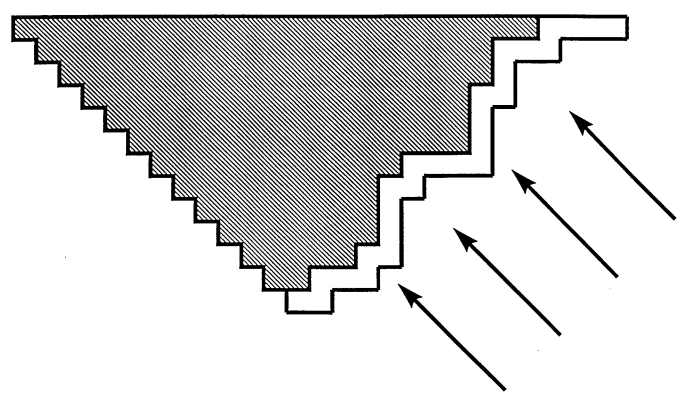

FIGURE 9.1.

Theorem 9.1. $\mathbb{E}(\mathbf{T}) \simeq \overline{\mathbb{O}}(\mathbf{T}) / \mathbb{O}(\mathbf{T})$.

Proof. By definition, $\mathbb{O}(\mathbf{T})$ is the kernel of $\nu$. This implies the result.

Now we can show that in the case of ribbon tiles most of the invariants cannot be derived by the extended coloring arguments. We shall give a complete description of the torsion group $\mathbb{E}\left(\mathbf{T}_{n}\right)$ and compare it with the previously computed tile counting group $\mathbb{G}\left(\mathbf{T}_{n}\right) \supset \mathbb{E}\left(\mathbf{T}_{n}\right)$.

Definition 9.2. Let $\mathbf{T}_{n}$ be a set of ribbon tiles. A map $f_{\mathbf{V}}: \mathbf{T}_{n} \rightarrow \mathbb{Z}_{n}$ defined as

$$
f_{\mathbf{v}}\left(\varepsilon_{1}, \ldots, \varepsilon_{n-1}\right)=\sum_{i=1}^{n-1} \cdot \varepsilon_{i} \quad(\bmod n)
$$

is called the shade invariant.

An easy geometric interpretation of $f_{\mathbf{v}}$ is given in Figure 9.1. Imagine there is a wall behind our ribbon tile $\tau$, and the light is coming from the southeast. Then $f_{\mathbf{V}}(\tau)$ is equal to the shaded area modulo $n$.

First, observe that $f_{\mathbf{v}}$ is a tile invariant. Indeed, if $n$ is odd we have

$$
f_{\mathbf{v}}=f_{1}+2 f_{2}+\cdots+m f_{m} \quad(\bmod n)
$$

where $f_{i}$ is the $i$-convexity invariant and $n=2 m+1$. Analogously, if $n$ is even we have

$$
f_{\mathbf{v}}=f_{1}+2 f_{2}+\cdots+(m-1) f_{m-1}+\left.f_{*}\right|^{\mathbb{Z}_{n}}(\bmod n),
$$

where $n=2 m$ and $g=\left.f_{*}\right|^{\mathbb{Z}_{n}} \bmod n$ is a parity invariant lifted to $\mathbb{Z}_{2 m}$ :

$$
g\left(\varepsilon_{1}, \ldots, \varepsilon_{n-1}\right)=m \varepsilon_{m} \quad(\bmod 2 m) .
$$

This gives $f_{\mathbf{V}} \in \mathbb{G}(\mathbf{T})$. Let us show that $f_{\boldsymbol{\nabla}} \in \mathbb{E}(\mathbf{T})$, i.e. that the shade invariant can be obtained by the extended coloring argument. Indeed, consider a coloring map $f: \mathcal{R} \rightarrow \mathbb{Z}$, defined by $f(i, j)=i \bmod n$. It is easy to see that $\nu(f)=f_{\mathbf{V}}$ and therefore $f_{\mathbf{v}} \in \mathbb{E}\left(\mathbf{T}_{n}\right)$. Analogously, the area invariant $f_{0}=\nu(g) \in \mathbb{E}\left(\mathbf{T}_{n}\right)$, where $g: \mathcal{R} \rightarrow \mathbb{Z}$ is a coloring map defined by

$$
f(i, j)= \begin{cases}1, i-j=0 & \bmod n, \\ 0, i-j \neq 0 & \bmod n .\end{cases}
$$

We claim that except for $f_{0}$ and $f_{\mathbf{v}}$ and their linear combinations, no other nontrivial invariant can be obtained by the extended coloring argument. 
Theorem 9.3. Let $\mathbf{T}_{n}$ be a set of ribbon tiles, $f_{0}$ the area invariant and $f_{\mathbf{v}}$ the shade invariant. Then $\mathbb{E}\left(\mathbf{T}_{n}\right) \simeq \mathbb{Z} \times \mathbb{Z}_{n}$, and the maps $f_{0}$, f form an independent basis of invariants.

Proof. We already showed that $f_{0}, f_{\mathbf{v}} \in \mathbb{E}\left(\mathbf{T}_{n}\right)$. Since they are independent, together they generate $\mathbb{Z} \times \mathbb{Z}_{n}$.

In the other direction, recall the computation of the coloring group $\mathbb{O}\left(\mathbf{T}_{n}\right) \simeq$ $\mathbb{Z}^{n-1}$ given in Theorem 8.2. Let us compute $\overline{\mathbb{O}}\left(\mathbf{T}_{n}\right)$. By Theorem 9.1 this is all we need to find $\mathbb{E}(\mathbf{T})$.

Denote $\tau_{0}=\mathbf{0 0} \ldots \mathbf{0}, \tau_{1}=\mathbf{1 1} \ldots \mathbf{1}$ and $\tau_{2}=\mathbf{1 0} \ldots \mathbf{0}$. Let us find all coloring maps $f: \mathcal{R} \rightarrow \mathfrak{G}$ (see the proof of Theorem 8.1), $f(i, j)=x_{i, j}$ such that the sums of squares in translations of $\tau_{0-2}$ are constant. Note here that a priori $\nu(f)$ does not have to be a tile invariant, since we do not check the relations for other ribbon tiles.

We claim that $f$ is determined by values $x_{1,1}, x_{1,2}, \ldots, x_{1, n}$ and $x_{2,1}$. Indeed, translations of $\tau_{0}$ and $\tau_{1}$ give us $x_{i, j}=x_{i \pm n, j}=x_{i, j \pm n}$. Now, given $x_{i, j}, \ldots x_{i, j+n-2}$ and the value $f\left(\tau_{2}\right)=x_{1,1}+\cdots+x_{1, n-1}+x_{2,1}$, we get

$$
x_{i+1, j}=f\left(\tau_{2}\right)-x_{i, j}-\cdots-x_{i, j+n-2} .
$$

Therefore, given $x_{1,1}, \ldots, x_{1, n}$ and $x_{2,1}$, we first determine $x_{1, j}$, for all $j \in \mathbb{Z}$, then $x_{2, j}$, then $x_{3, j}$, etc. For the negative rows use $x_{i, j}=x_{i+n, j}$. This proves the claim.

Now, by taking a quotient $\overline{\mathbb{O}}\left(\mathbf{T}_{n}\right) / \mathbb{O}\left(\mathbf{T}_{n}\right)$ we can make all values $x_{1,1}, x_{1,2}$, $\ldots, x_{1, n-1}$ zero (see the proof of Theorem 8.2). Let $x_{1, n}=a, x_{2,1}=a+z$. The computations above give us $x_{i+1, j}=a+i z$ and $n z=0$. Therefore $f=a \cdot f_{0}+z \cdot f_{\mathbf{V}}$, and $f_{0}, f_{\mathbf{v}}$ generate the whole torsion group $\mathbb{E}\left(T_{n}\right)$.

As a corollary, from Theorem 9.3 we immediately get Theorem 1.8. As we noted in the introduction, another way to prove Theorem 1.8 would be to find a tileable region $\Gamma \in \mathcal{R}_{\mathbf{T}_{n}} \backslash \mathcal{R}_{r c}, n \geq 3$, such that $f_{\bullet}$ is not constant on the set $\mathcal{S}\left(\Gamma, \mathbf{T}_{n}\right)$ of ribbon tilings. When $n=3$ one such example is shown in Figure 9.2. The value of $f_{\bullet}$ is 1 on the first tiling and 0 on the second tiling. Note also that such a region $\Gamma$ probably must have at least one hole inside (see Conjecture 10.1 in the next section).
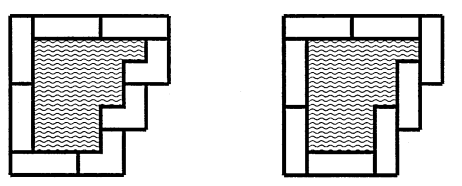

FIGURE 9.2.

\section{Conclusion}

Let us summarize the results in the paper and compare them with open questions.

The main result of the paper is a description of the tile counting group for a set of ribbon tiles. Note, however that we only considered row convex or column convex regions (which probably include all the interesting ones). However, Conway and Lagarias in CL were able to prove that for $n=3$ the map $f_{1}$ is an invariant for all simply connected regions. Recently Muchnik and the author in $\mathrm{MP}$ used a similar 
technique to show that for $n=4$ the maps $f_{1}$ and $f_{*}$ are invariants for all simply connected regions. All the available evidence points to the following conjecture $*$

Conjecture 10.1. The $i$-convexity and parity maps are the group invariants for all simply connected regions.

The major point of our proof is Theorem 1.6, which claims that there is a finite set of moves which can change any tiling of a given Young shape region to any other tiling. This result has been generalized by the author for any skew shape (see $\mathrm{Pa}]$ ). In fact we believe in the following conjecture.

Conjecture 10.2. Let $\Gamma$ be any simply connected region tileable by $\mathbf{T}_{n}$. Then the graph $\Theta_{n}(\Gamma)$ is connected.

In other words, we claim that the moves defined in section 3 suffice. Of course, the results of section 4 imply that Conjecture 10.1 follows from Conjecture 10.2.

Let us move now to other sets of tiles. Unfortunately it is not always true that there exist a finite number of moves (or local replacement rules as they are also called). For example, let $\mathbf{T} \supset \mathbf{T}_{3}$ be the set of all trominoes (see [G] and Figure 10.1). There are infinitely many regions with exactly two tilings that are not local in any sense (see Figure 10.2). Even though there are no finite number of local moves, there still can be some invariants other than the area. Here is an example.

Let $\mathbf{T}$ be the set of four trominoes $\tau_{1}, \ldots, \tau_{4}$ in Figure 10.1. Let $\mathcal{B}=\mathcal{R}_{\mathbf{T}}$ be the set of all $\mathbf{T}$-tileable regions. Define maps $f_{1,2}, f_{2,3}: \mathbf{T} \rightarrow \mathbb{Z}_{3}$ as follows:

$$
\begin{array}{ll}
f_{1,2}\left(\tau_{1}\right)=f_{1,2}\left(\tau_{2}\right)=1, & f_{1,2}\left(\tau_{3}\right)=f_{1,2}\left(\tau_{4}\right)=0, \\
f_{2,3}\left(\tau_{2}\right)=f_{2,3}\left(\tau_{3}\right)=1, & f_{2,3}\left(\tau_{1}\right)=f_{2,3}\left(\tau_{4}\right)=0 .
\end{array}
$$

Theorem 10.3. Let $\mathbf{T}$ and $\mathcal{B}=\mathcal{R}_{\mathbf{T}}$ be as above, and let $f_{0}$ be an area invariant. Then tile counting group

$$
\mathbb{G}(\mathbf{T}, \mathcal{B}) \simeq \mathbb{Z} \times \mathbb{Z}_{3}^{2}
$$

and the maps $f_{0}, f_{1,2}, f_{2,3}$ form an independent basis of invariants.

Theorem 10.3 basically claims that there is one nontrivial tile invariant $f_{1,2}$, which can be stated as follows:

- For any convex region $\Gamma$ the number of times modulo 3 the tiles $\tau_{1}$ and $\tau_{2}$ occur in a tiling of $\Gamma$ depends only on $\Gamma$.

Theorem 10.3 also claims that rotations of $f_{1,2}$ and the area invariant generate the whole tile invariant group.

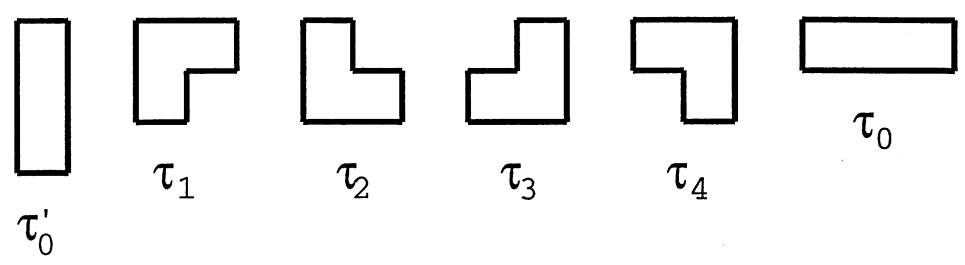

FiguRe 10.1.

\footnotetext{
${ }^{*}$ Conjecture 10.1 was recently established by C. Moore and the author (July 2000).
} 


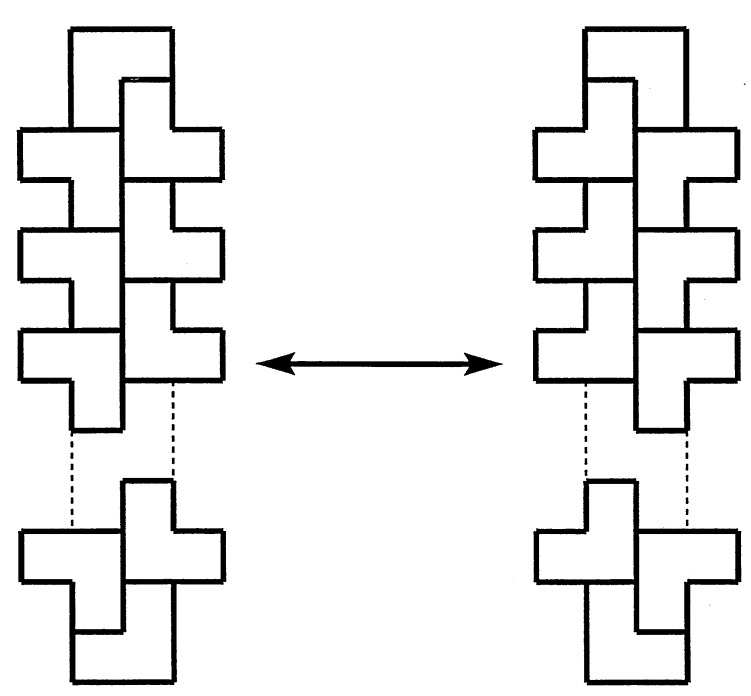

FiguRe 10.2.
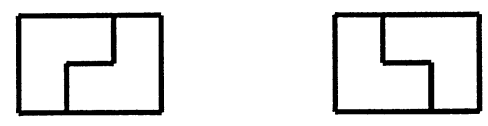

Figure 10.3.

Proof of Theorem 10.3. The idea is similar to the one used in section 6. By definition $\mathbb{G}(\mathbf{T} ; \mathcal{B})=\mathbb{Z}^{\mathbf{T}} / I$, where $I$ is the linear span or relations obtained from different tilings of the same region. Thus having enough relations that generate $I^{\prime} \subset I$ will imply that $\mathbb{G}(\mathbf{T} ; \mathcal{B}) \subset \mathbb{Z}^{\mathbf{T}} / I^{\prime}$. In this particular case two types of relations will suffice.

First, there is a relation obtained from the two tilings of $[2 \times 3]$ (see Figure 10.3):

$$
\tau_{1}+\tau_{3}=\tau_{2}+\tau_{4} .
$$

Then there is another relation which comes from the two tilings in Figure 10.4:

$$
4 \cdot \tau_{4}+\tau_{2}=4 \cdot \tau_{3}+\tau_{1} .
$$

In combination with the first relation and rotations, this gives $3 \cdot \tau_{1}=\cdots=3 \cdot \tau_{4}$. Simple further computations show that the maps $f_{0}, f_{1,2}, f_{2,3}$ are independent and generate the whole tile counting group $\mathbb{G}\left(\mathbf{T}, \mathcal{R}_{\mathbf{T}}\right)$. Therefore we have $\mathbb{G}\left(\mathbf{T}, \mathcal{R}_{\mathbf{T}}\right) \subset$ $\mathbb{Z} \times \mathbb{Z}_{3}^{2}$.

Now it remains to prove that $f_{1,2}$ is indeed an invariant. This in turn would imply that $f_{1,2}$ is an invariant, and prove the theorem. This can be done by the tile extended coloring argument.

Let $g: \mathcal{R} \rightarrow \mathbb{Z}_{3}$ be a coloring map defined by $g(i, j)=j-i(\bmod 3)$. Observe that $g \in \overline{\mathbb{O}}(\mathbf{T})$. Compute the corresponding tile invariant $f=\nu(g): \mathcal{R}_{\mathbf{T}} \rightarrow \mathbb{Z}_{3}$. We have

$$
f\left(\tau_{1}\right)=f\left(\tau_{3}\right)=0, f\left(\tau_{2}\right)=-1, f\left(\tau_{2}\right)=1 \quad \bmod 3 .
$$



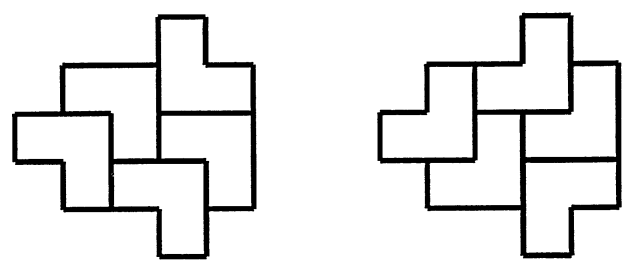

Figure 10.4 .
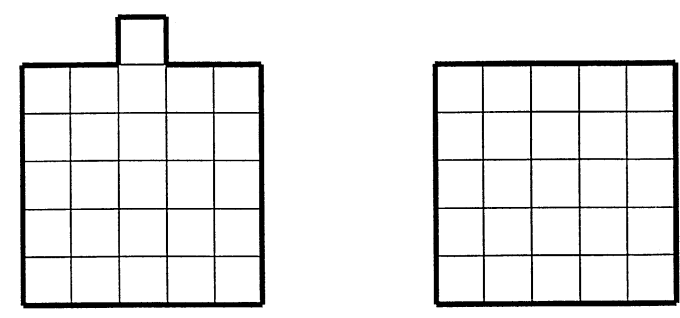

FiguRE 10.5.

By the symmetry, we also have another tile invariant $f^{\prime}: \mathcal{R}_{\mathbf{T}} \rightarrow \mathbb{Z}_{3}$, given by

$$
f^{\prime}\left(\tau_{2}\right)=f^{\prime}\left(\tau_{4}\right)=0, f^{\prime}\left(\tau_{1}\right)=-1, f^{\prime}\left(\tau_{3}\right)=1 \quad \bmod 3 .
$$

From this we get

$$
f_{1,2}=f+f^{\prime}-f_{0} \quad \bmod 3 .
$$

Therefore both $f_{1,2}$ and $f_{2,3}$ are $\mathbf{T}$-invariants. This finishes the proof.

Note that in the proof of Theorem 10.3 we used nothing but coloring arguments. Of course, with a smaller set of tileable regions, when the tile counting group becomes bigger, this would be impossible. That was the case with ribbon tiles. Indeed, in section 9 we showed that the height invariant cannot be extended to the set of all tileable regions, so the tile counting group $\mathbb{G}\left(\mathbf{T}_{n}, \mathcal{R}_{\mathbf{T}_{n}}\right) \subsetneq \mathbb{G}\left(\mathbf{T}_{n}, \mathcal{B}_{r c}\right)$. Thus finding the tile counting group is probably hard in general unless all invariants follow from the extended coloring arguments.

Even in the case of all T-tileable regions it is still possible to have invariants which do not follow from any extended coloring arguments. Indeed, consider the set $\mathbf{T}$ of two tiles shown in Figure 10.5. It is easy to see that any region either is untileable or has a unique tiling. On the other hand, extended coloring arguments can prove only the area invariant. Note that in this case every region has a signed tiling.

We believe that having $\mathbb{E}(\mathbf{T}) \simeq \mathbb{G}\left(\mathbf{T}, \mathcal{R}_{\mathbf{T}}\right)$, is a rather rare event. However it might occur for certain nice sets of tiles such as finite sets of rectangles. Without making a precise conjecture, let us state the following problem.

Problem 10.4. Let $\mathbf{T}$ be a finite set of rectangles, and let $\mathcal{B}$ be the set of convex regions tileable by $\mathbf{T}$. Find tile invariant group $\mathbb{G}(\mathbf{T} ; \mathcal{B})$ and the torsion group $\mathbb{E}(\mathbf{T})$. 
For example, let $\mathbf{T}$ consist of two rectangles $[2 \times 1]$ and $[1 \times 3]$. It is not hard to see that $\mathbb{G}(\mathbf{T} ; \mathcal{B}) \simeq \mathbb{E}(\mathbf{T}) \simeq \mathbb{Z} \times \mathbb{Z}_{5}$. There is some recent literature relevant to the problem (see $\mathrm{Ke}]$ for details).

In this paper we always considered tiles to be identical if they can be obtained by translation. Following some recent literature (see e.g. $[\overline{\mathrm{Pr}}]$ ), one can try to distinguish between ribbon tiles with different starting points. It seems to us that at least theoretically the whole analysis of the paper can be generalized for this case, though some computations may become complicated. We challenge the reader to find tileability applications of these generalizations.

Our proof of the main theorem was based on an ad hoc method which probably cannot be generalized in full for other sets of tiles. The heart of the proof is the rim hook bijection. There are shifted, tree, and skew analogs of this bijection (see [FS] and $[\mathrm{Pa}]$ ), but they all can be reduced to the original bijection in one way or another.

Problem 10.5. Find a three-dimensional analog of the rim hook bijection.

Of course, there are infinitely many open questions and problems, but the reader is probably too tired already to be bothered by whatever is left.

\section{ACKNOWLEDGMENTS}

I would like to express my deep gratitude to Jim Propp for interest in my work, for encouragement, for reading the first draft of this paper, and for useful suggestions. Conversations with Richard Stanley and his comments were very helpful. Nathan Lulov asked me the initial question which later became Theorem 1.8.

\section{REFERENCES}

[BW] A. Bjorner, M. Wachs, Generalized quotients in Coxeter groups., Trans. Amer Math. Soc. 308 (1988), 1-37. MR 89c:05012

[BK] A. Berenstein, A. Kirillov, Groups generated by involutions, Gelfand-Tsetlin patterns, and combinatorics of Young tableaux, St. Petersburg Math. J. 7 (1996), 77-127. MR 96e:05178

[CEP] H. Cohn, N. Elkies, J. Propp, Local statistics for random domino tilings of the Aztec diamond, Duke Math. J. 85 (1996), 117-166. MR 97k:52026

[CL] J. H. Conway, J. C. Lagarias, Tilings with polyominoes and combinatorial group theory, J. Comb. Theory, Ser. A 53 (1990), 183-208. MR 91a:05030

[EKLP] N. Elkies, G. Kuperberg, M. Larsen and J. Propp, Alternating sign matrices and domino tilings. I, II, J. Alg. Comb. 1 (1992), 111-132, 219-234. MR 94f:52035, MR 94f:52036

[FS] S. Fomin, D. Stanton, Rim hook lattices, St. Petersburg Math. J. 9 (1998), 1007-1016. MR 99c:05202

[GJ] M. Garey, D. S. Johnson, Computers and Intractability: A Guide to the Theory of NP-completeness, Freeman, San Francisco, CA, 1979. MR 80g:68056

[G] S. Golomb, Polyominoes, Scribners, New York, 1965. MR 95k:00006 (later ed.)

[JK] G. James, A. Kerber, The Representation Theory of the Symmetric Group, AddisonWesley, Reading, MA, 1981. MR 83k:20003

[Ka] P. W. Kastelyn, The statistics of dimers on a lattice. I. The number of dimer arrangements on a quadratic lattice, Physica 27 (1961), 1209-1225.

[Ke] R. Kenyon, A note on tiling with integer-sided rectangles, J. Combin. Theory, Ser. A 74 (1996), 321-332. MR 97c:52045

[M] I. G. Macdonald, Symmetric Functions and Hall Polynomials, Oxford University Press, London, 1979. MR 84g:05003

[MP] R. Muchnik, I. Pak, On tilings by ribbon tetrominoes, J. Combin. Theory, Ser. A 88 (1999), 199-193. CMP 2000:01

[Pa] I. Pak, A generalization of the rim hook bijection for skew shapes, preprint, 1997. 
[Pr] J. Propp, A pedestrian approach to a method of Conway, or, A tale of two cities, Math. Mag. 70 (1997), 327-340. MR 98m:52031

[R] G. de B. Robinson, Representation Theory of the Symmetric Group, Edinburgh University Press and Univ. of Toronto Press, 1961. MR 23:A3182

[S] R. P. Stanley, Enumerative Combinatorics. Vol. 2, Cambridge Univ. Press, 1999. CMP 99:09

[SW] D. Stanton, D. White, A Schensted algorithm for rim hook tableaux, J. Comb. Theory, Ser. A 40 (1985), 211-247. MR 87c:05014

[TF] H. N. V. Temperley, M. E. Fisher, Dimer problem in statistical mechanics - An exact result, Philos. Mag. 6 (1961), 1061-1063. MR 24:B2436

[T] W. Thurston, Conway's tiling group, Amer. Math. Monthly 97 (1990), 757-773. MR91k:52028

Department of Mathematics, Yale University, New Haven, Connecticut 06520-8283

E-mail address: paki@math.yale.edu

Current address: Department of Mathematics, MIT, Cambridge, Massachusetts 02139

E-mail address: paki@math.mit.edu 